\title{
THE GEOMETRY OF CYCLIC HYPERBOLIC POLYGONS
}

\author{
JASON DEBLOIS
}

\begin{abstract}
We will call a hyperbolic polygon cyclic, horocyclic, or equidistant if its vertices lie on a metric circle, a horocycle, or a component of the equidistant locus to a hyperbolic geodesic, respectively. Such convex $n$-gons are parametrized by the subspaces of $\left(\mathbb{R}^{+}\right)^{n}$ that contain their side length collections, and area and circumcircle or "collar" radius determine symmetric, smooth functions on these spaces. We give formulas for and bounds on the derivatives of these functions and make some observations on their behavior. Notably, the monotonicity properties of area and circumcircle radius exhibit qualitative differences on the collection of centered vs non-centered cyclic polygons, where a cyclic polygon is centered if it contains the center of its circumcircle in its interior.
\end{abstract}

An $n$-tuple $\left(d_{0}, \ldots, d_{n-1}\right)$ of positive real numbers is the side length collection of a compact, convex $n$-gon $P$ in the hyperbolic plane, $\mathbb{H}^{2}$, that is cyclic or horocyclic, or has all vertices equidistant from a fixed geodesic, as long as

$$
d_{i} \leq \sum_{j \neq i} d_{j}, \quad \text { for each } i .
$$

Moreover, such a polygon, $P$, is uniquely prescribed up to orientationpreserving isometry of $\mathbb{H}^{2}$ by this $n$-tuple up to cyclic permutation. It is cyclic if and only if, for each $i$,

$$
\sinh \left(\frac{d_{i}}{2}\right)<\sum_{j \neq i} \sinh \left(\frac{d_{j}}{2}\right)
$$

and $P$ is horocyclic if and only if, for some $i, d_{i}$ is the sum of the other side lengths.

2010 AMS Mathematics subject classification. Primary 51M09.

Keywords and phrases. Cyclic, polygon, convex, hyperbolic.

Partially supported by grant NSF DMS-1240329.

Received by the editors on July 18, 2014. 
This fact seems to have been independently rediscovered several times. It was recorded by the late Stothers [13], then by Schlenker (see [12, page 2175]) as part of a larger project. Schlenker's results on cyclic polygons were reproved by Walter $[\mathbf{1 5}, \mathbf{1 6}]$. Propositions $1.14,3.4$ and 4.6 of this paper combine for a self-contained case-by-case proof. Our approach is standard, compare, e.g., Robbins [11] (in the Euclidean setting). All aspects are elementary, but the cyclic case is somewhat subtle; see the discussion at the beginning of Section 1 .

Our main goal is to make some observations on the qualitative behavior of area and circumcircle radius, thought of as functions on a subset $\mathcal{A C}_{n}$ of $\left(\mathbb{R}^{+}\right)^{n}$ that parametrizes cyclic $n$-gons by side length, for each $n \geq 3$. We also record relatively simple formulas for their derivatives and give useful bounds in some cases. Letting $\mathbf{d}=\left(d_{0}, \ldots, d_{n-1}\right) \in\left(\mathbb{R}^{+}\right)^{n}$, in view of the facts above, we define:

$\mathcal{A C}_{n}=\left\{\mathbf{d} \mid \sinh \left(\frac{d_{i}}{2}\right)<\sum_{j \neq i} \sinh \left(\frac{d_{j}}{2}\right)\right.$ for each $\left.i \in\{0, \ldots, n-1\}\right\}$.

One of our key themes is that the functions on $\mathcal{A C}_{n}$ determined by area and circumcircle radius exhibit qualitatively different behaviors on centered versus non-centered cyclic $n$-gons. We say a cyclic polygon is centered if it contains its circumcircle center in its interior.

The collection $\mathcal{C}_{n} \subset \mathcal{A C}_{n}$ defined below parametrizes centered $n$ gons. Here, for $J \geq d / 2$, let $\theta(d, J)=2 \sin ^{-1}\left(\sinh \left(d_{i} / 2\right) / \sinh J\right)$ :

$$
\mathcal{C}_{n}=\left\{\mathbf{d} \mid \sum_{i=0}^{n-1} \theta\left(d_{i}, \frac{D}{2}\right)>2 \pi, \text { where } D=\max \left\{d_{i}\right\}_{i=0}^{n-1}\right\} .
$$

The qualitative differences we mentioned above are visible in the following bounds:

Proposition 1.20. For $n \geq 3$, the function $J: \mathcal{A C}_{n} \rightarrow \mathbb{R}^{+}$that records the circumcircle radius is smooth and symmetric. For $\mathbf{d} \in \mathcal{A C}_{n}$,

$$
\begin{cases}0<\left(\partial J / \partial d_{i}\right)(\mathbf{d})<1 / 2 & \text { if } \mathbf{d} \in \mathcal{C}_{n}, \text { for any } i, \\ \left(\partial J / \partial d_{i_{0}}\right)(\mathbf{d})>1 / 2 & \text { if } \mathbf{d} \in \mathcal{A C}_{n}-\left(\mathcal{C}_{n} \cup \mathcal{B C}_{n}\right) \\ & \text { and } d_{i_{0}}=\max \left\{d_{i}\right\}_{i=0}^{n-1}, \\ \left(\partial J / \partial d_{j}\right)(\mathbf{d})<0 & \text { if } \mathbf{d} \in \mathcal{A C}_{n}-\left(\mathcal{C}_{n} \cup \mathcal{B C} \mathcal{C}_{n}\right) \\ & \text { and } d_{j} \neq \max \left\{d_{i}\right\}_{i=0}^{n-1} .\end{cases}
$$


If $\mathbf{d}=\left(d_{0}, \ldots, d_{n-1}\right)$ and $d_{i}>d_{j}$, then

$$
\left|\frac{\partial J}{\partial d_{i}}(\mathbf{d})\right|>\left|\frac{\partial J}{\partial d_{j}}(\mathbf{d})\right| \text {. }
$$

The subspace $\mathcal{B C}_{n}$ of $\mathcal{A C}_{n}$ referenced above is the frontier of $\mathcal{C}_{n}$ in $\mathcal{A C}_{n}$, a codimension-one submanfold that parametrizes semicyclic $n$-gons: those whose longest edge is a diameter of their circumcircle. (See Propositions 1.17 and 1.18. The term "semicyclic" is due to Maley, Robbins and Roskies in the Euclidean setting [6].) Values of the $\partial J / \partial d_{i}$ on $\mathcal{B C}_{n}$ are thus determined by continuity and the formulas above.

We also give a Schläfli-type formula on areas of cyclic $n$-gons. Whereas the classical Schläfli formula expresses change in the area of a first-order deformation of polygons in terms of angle variations, ours is in terms of side length. And, since cyclic polygons are parametrized by their side length collections, we can simply record partial derivatives.

Proposition 2.3. For $n \geq 3$, the function $D_{0}: \mathcal{A C}_{n} \rightarrow \mathbb{R}^{+}$that records hyperbolic area is smooth and symmetric. Its partial derivative $\partial D_{0} / \partial d_{i}$ with respect to $d_{i}$ is given at $\mathbf{d}=\left(d_{0}, \ldots, d_{n-1}\right) \in \mathcal{A C}_{n}$ by:

$$
\begin{cases}-\sqrt{\left[1 / \cosh ^{2}\left(d_{i} / 2\right)\right]-\left[1 / \cosh ^{2} J(\mathbf{d})\right]} & \text { if } \mathbf{d} \in \mathcal{A C}_{n}-\mathcal{C}_{n} \text { and } \\ & d_{i}=\max \left\{d_{j}\right\}_{j=0}^{n-1}, \\ \sqrt{\left[1 / \cosh ^{2}\left(d_{i} / 2\right)\right]-\left[1 / \cosh ^{2} J(\mathbf{d})\right]} & \text { otherwise. }\end{cases}
$$

Note that the circumcircle radius of a semicyclic $n$-gon is half its longest side length, so the two formulas above are compatible with continuous partial derivatives of $D_{0}$; in particular, the first is 0 everywhere on $\mathcal{B C}_{n}$. One consequence of Proposition 2.3 is that the area of centered and semicyclic $n$-gons is monotonic in their side lengths.

Corollary 2.4. For $n \geq 3, \mathbf{d}=\left(d_{0}, \ldots, d_{n-1}\right)$ and $\mathbf{d}^{\prime}=\left(d_{0}^{\prime}, \ldots, d_{n-1}^{\prime}\right)$ in $\mathcal{C}_{n} \cup \mathcal{B C}_{n}$ if, after a permutation $d_{i} \leq d_{i}^{\prime}$ for all $i$, and $d_{i}<d_{i}^{\prime}$ for some $i$, then $D_{0}(\mathbf{d})<D_{0}\left(\mathbf{d}^{\prime}\right)$.

Section 3 extends the above considerations to horocyclic polygons in $\mathbb{H}^{2}$. We parametrize horocyclic $n$-gons by a codimension- 1 submanifold 
$\mathcal{H C}_{n}$ of $(0, \infty)^{n}$, which is the frontier there of $\mathcal{A C}_{n}$. The function $J: \mathcal{A C}_{n} \rightarrow \mathbb{R}^{+}$blows up approaching $\mathcal{H C}_{n}$, see Proposition 3.6, suggesting the geometric interpretation that horocyclic $n$-gons are limits of sequences of cyclic $n$-gons whose circumcircle radii go to infinity.

Section 3 also describes horocyclic ideal polygons, which have all vertices on a horocycle except one at its ideal point, see Definition 3.3. These are natural limits for families of cyclic polygons with certain edge lengths going to infinity, and the area function $D_{0}$ extends continuously to their parameter space $\mathcal{H} \mathcal{I}_{n}$. See Propositions 3.7 and 3.9.

Below, again taking $\mathbf{d}=\left(d_{0}, \ldots, d_{n-1}\right) \in\left(\mathbb{R}^{+}\right)^{n}$, we define:

$$
\begin{aligned}
\mathcal{E}_{n}=\left\{\mathbf{d} \mid \text { there exists } i_{0}\right. \text { with } & \sinh \left(d_{i_{0}} / 2\right) \\
& \left.>\sum_{i \neq i_{0}} \sinh \left(d_{i} / 2\right) \text { but } d_{i_{0}} \leq \sum_{i \neq i_{0}} d_{i}\right\} .
\end{aligned}
$$

In Section 4, it is shown that $\mathcal{E}_{n}$ parametrizes equidistant polygons. Like $\mathcal{A C}_{n}$, it has nonempty interior in $(0, \infty)^{n}$, and its closure there intersects that of $\mathcal{A C}_{n}$ in $\mathcal{H C}_{n}$.

There is a collar radius function $J: \mathcal{E}_{n} \rightarrow[0, \infty)$ analogous to the circumcircle radius function on $\mathcal{A C}_{n}$, with similar behavior, see Proposition 4.7. The area function $D_{0}$ extends continuously to $\mathcal{A C}_{n} \cup$ $\mathcal{H C}_{n} \cup \mathcal{E}_{n}$ by Propositions 3.7 and 4.9. Proposition 4.9 also gives a Schläfli-type formula similar to the one above for $D_{0}$ on $\mathcal{E}_{n}$. Combining Propositions 2.3 and 4.9 with Schlenker's results yields:

Corollary 4.11. For fixed positive real numbers $d_{1}, \ldots, d_{n-1}$, among all hyperbolic $n$-gons with $n-1$ sides of these lengths, the area is maximized by the semicyclic ones with final side length greater than $\max \left\{d_{i}\right\}$.

The analogous result for Euclidean polygons is mentioned by Maley, Robbins and Roskies [6, page 672]. We do not know a reference for a proof.

Finally, in Section 5, we describe how cyclic $n$-gons degenerate to cyclic $m$-gons (for $m<n$ ) as some side lengths approach 0 . In terms of 
our parameter spaces, the frontier of $\mathcal{A C}_{n} \cup \mathcal{H C}_{n}$ in $[0, \infty)^{n}$ is a union of copies of $\mathcal{A C}_{m} \cup \mathcal{H C}_{m}$, for $3 \leq m<n$, and a degenerate part (see Lemma 5.1). The main results are that the radius and area functions on $\mathcal{A C}_{n}$ extend continuously to those on $\mathcal{A C}_{m}$, see Lemma 5.3 and Corollary 5.5, respectively.

The results here are an important tool in [4], on Delaunay tessellations of hyperbolic surfaces. The 2-cells of such a decomposition are cyclic, horocyclic or equidistant polygons, see [3].

In the Euclidean setting, a body of work giving explicit formulas for area and circumcircle radius reaches back to classical times. Heron's formula, recorded circa A.D. 60, and Brahmagupta's formula from the seventh century give the areas of cyclic Euclidean triangles and quadrilaterals, respectively, as functions of their side lengths. In 1828, Möbius gave formulas for circumcircle radius [8]. More recently, Robbins gave a series of conjectures on a polynomial relation extending the Heron and Brahmagupta formulas to arbitrary $n$-gons [11], which were subsequently proved in the independent work of several authors, see e.g., [9].

A hyperbolic version of Heron's formula was only recorded in 1969 by Bilinski [1], then independently rediscovered by Stothers. Even more recently, Mednykh found a hyperbolic Brahmagupta formula [7]. We know of no other literature on cyclic hyperbolic polygons beyond what has been cited above. Conversely, we are not aware of recorded Euclidean analogs to our results.

1. Calculus (and some geometry). A straightforward method of continuity approach to proving existence of cyclic polygons with given side lengths is neatly sketched by Robbins in the Euclidean setting [11, page 526]:

Imagine a circle of variable radius and let us try to inscribe a polygon with sides of the given lengths in the circle by picking an arbitrary starting point and laying out the edges, one at a time, with the given lengths. When the radius is too large, we will not reach the starting point when we have used up all the sides. As we decrease the radius there will come a time when we return exactly to our starting point. 

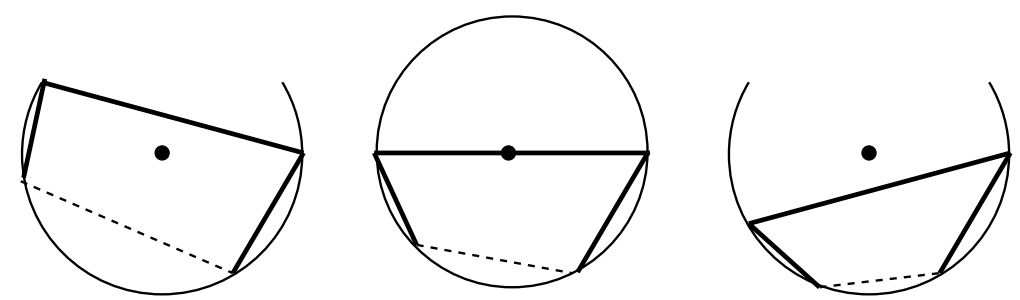

Figure 1. From left to right, circle radius decreases then increases, but distance between endpoints of the union of edges continues to decrease.

This only works given two assumptions (both pointed out by Robbins). First, that all side lengths are nearly equal, and second, that all edges go in the same direction, that is, each edge joins its initial vertex $x_{i}$ to the possibility for $x_{i+1}$ which is closer on the circle to $x_{i}$ in the direction of some fixed orientation.

If one drops the first assumption, but keeps the second, then sometimes the circle radius can decrease as far as possible without the starting point being reached. But, on dropping the second assumption, uniqueness can fail since, if the circle radius is $J$ and $d<2 J$, then there are two possible edges of length $d$ with any given initial point.

However, for convex polygons, it is not hard to see that all edges except possibly the longest must go in the same direction, and Robbins' strategy can be successfully modified as follows:

Having laid out all edges in the same direction, if on decreasing the radius as far as we can (to half the length of the longest side) we have not yet returned to our starting point then re-inflate the circle, now forcing the longest edge to go in the opposite direction from the others. As we increase the radius there will come a time when we return exactly to our starting point (assuming the edge lengths satisfy the proper inequality).

See Figure 1. Below we prove existence and uniqueness of convex cyclic hyperbolic $n$-gons by formalizing a slight variation on this approach. Rather than laying out all $n$ edges, we lay out $n-1$, and we vary the circle radius until the distance between start and end points 
equals the length of the omitted edge. Proceeding in this way helps with the calculus.

Before we begin, we note that, in [11], Robbins considers Euclidean cyclic $n$-gons with no convexity requirement whereupon uniqueness does indeed fail, see [11, Diagram 1]. In fact, the extent of its failure determines the degree of the "generalized Heron polynomials" that are the main objects of study in [11].

1.1. Existence and uniqueness. Let us begin by specifying a model of the hyperbolic plane and recalling a few basic facts, which can be found in, e.g., [10].

Definition 1.1. The upper half-plane model of the hyperbolic plane is the open subset,

$$
\mathbb{H}^{2} \doteq\{z \in \mathbb{C} \mid \Im z>0\}
$$

of $\mathbb{C}$, which inherits the standard orientation and is endowed with the Riemannian metric $\left(d x^{2}+d y^{2}\right) / y^{2}$ (identifying $\mathbb{C}$ with $\mathbb{R}^{2}$ ). For $x$ and $x^{\prime}$ in $\mathbb{H}^{2}$ let $\operatorname{dist}\left(x, x^{\prime}\right)$ refer to the distance from $x$ to $x^{\prime}$ in the resulting path metric.

The orientation-preserving isometry group of $\mathbb{H}^{2}$ is $\mathrm{PSL}_{2}(\mathbb{R})$, acting by Möbius transformations. This action is transitive on $\mathbb{H}^{2}$, hence also on hyperbolic circles of fixed radius. Each hyperbolic circle is an Euclidean circle contained in $\mathbb{H}^{2}$, alalthough its hyperbolic and Euclidean center and radius do not coincide.

Definition 1.2. For points $x$ and $y$ on a circle $C$, the counterclockwise arc $[x, y]$ from $x$ to $y$ is the arc of $C$ bounded by $x$ and $y$ that points from $x$ to $y$ in the counterclockwise orientation on $C$. The angle from $x$ to $y$ is the angle subtended by $[x, y]$ at the center of $C$. We will say a collection $\left\{x_{0}, \ldots, x_{n-1}\right\} \subset C$ is cyclically ordered if, for all $i>0$, $\left(x_{i-1}, x_{i}\right]$ contains no $x_{j}$ for $j \neq i$.

A (convex) cyclic $n$-gon with side length collection $\left(d_{0}, \ldots, d_{n-1}\right) \in$ $(0, \infty)^{n}$ is a cyclically ordered collection $\left\{x_{0}, \ldots, x_{n-1}\right\}$ on a fixed hyperbolic circle, called the circumcircle, such that $\operatorname{dist}\left(x_{i-1}, x_{i}\right)=d_{i}$ for each $i>0$ and $\operatorname{dist}\left(x_{0}, x_{n-1}\right)=d_{0}$. An isometry from a cyclic $n$-gon $\left\{x_{0}^{\prime}, \ldots, x_{n-1}^{\prime}\right\}$ to $\left\{x_{0}, \ldots, x_{n-1}\right\}$ is an isometry of $\mathbb{H}^{2}$ that takes $x_{i}^{\prime}$ to $x_{\sigma(i)}$ for each $i$, where $\sigma$ is a fixed cyclic permutation. 
Remark 1.3. If an isometry takes $x_{i}^{\prime}$ to $x_{\sigma(i)}$ for each $i$, where $\sigma$ is a fixed cyclic permutation, then also $d_{i}^{\prime}=d_{\sigma(i)}$ for each $i$. In particular, cyclically relabeling a cyclic $n$-gon $\left\{x_{0}, \ldots, x_{n-1}\right\}$ by a cyclic permutation $\sigma$ produces a cyclic $n$-gon $\left\{x_{\sigma(0)}, \ldots, x_{\sigma(n-1)}\right\}$, isometric to the original via the identity map, with the side length collection that of the original relabeled by $\sigma$.

Hyperbolic trigonometry relates the distance between points on a circle to the angle from one to another as well as the circle radius. Here is the key function:

Lemma 1.4. For $d>0$ and $J \geq d / 2$, let $T$ be a hyperbolic triangle with two sides of length $J$ and a third of length $d$. Its interior angle at the vertex opposite the side of length $d$ is:

$$
\theta(d, J)=2 \sin ^{-1}(\sinh (d / 2) / \sinh J) \in[0, \pi] .
$$

This is a continuous function on $\{(d, J) \mid 0<d \leq 2 J\}$, smooth on its interior. For fixed $d>0, \theta(d, J)$ decreases in $J$ on $[d / 2, \infty)$, with $\theta(d, d / 2)=\pi, \lim _{J \rightarrow \infty} \theta(d, J)=0$ and

$$
\begin{aligned}
\partial \theta(d, J) / \partial J & =\frac{-2 \sinh (d / 2) \cosh J}{\sinh J \sqrt{\sinh ^{2} J-\sinh ^{2}(d / 2)}} \\
& =-2 \operatorname{coth} J \tan (\theta(d, J) / 2) .
\end{aligned}
$$

Proof. Applying the hyperbolic law of sines (see [10, Theorem 3.5.2]) to a triangle obtained from $T$ by dropping a perpendicular to the side with length $d$ from the vertex opposite it yields:

$$
\sinh (d / 2)=\sin (\theta(d, J) / 2) \sinh J .
$$

The formula for $\theta(d, J)$ follows. Continuity and smoothness of $\theta$ follow from the fact that the inverse sine is continuous on $[-1,1]$ and smooth on $(-1,1)$. By direct computation, $\theta(d, d / 2)=2 \sin ^{-1}(1)=\pi$ and, for fixed $d>0, \lim _{J \rightarrow \infty} \theta(d, J)=2 \sin ^{-1}(0)=0$. That $\theta(d, J)$ decreases in $J$ for fixed $d>0$ follows from the partial derivative computation, which is straightforward.

The result below describes the two ways to put a cyclic $n$-gon in a circle of fixed radius, specifying all but one of its side lengths. 
Proposition 1.5. For any $d_{1}, \ldots, d_{n-1} \in(0, \infty), J \geq \max \left\{d_{i} / 2\right\}$, and any hyperbolic circle $C$ of radius $J$, there exists $\left\{x_{0}, \ldots, x_{n-1}\right\} \subset C$ such that $\operatorname{dist}\left(x_{i-1}, x_{i}\right)=d_{i}$ for each $i>0$. One such collection has the angle $\theta_{i}$ from $x_{i-1}$ to $x_{i}$ equal to $\theta\left(d_{i}, J\right)$ for each $i>0$. For any fixed $i_{0}$, there is another such that $\theta_{i_{0}}=2 \pi-\theta\left(d_{i_{0}}, J\right)$, but $\theta_{i}=\theta\left(d_{i}, J\right)$ for each $i \neq i_{0}$. Each of these collections is determined, up to hyperbolic isometry, by the choice of $\theta_{i}$. The former is cyclically ordered if and only if

$$
\sum_{i=1}^{n-1} \theta\left(d_{i}, J\right)<2 \pi
$$

the latter if and only if

$$
\theta\left(d_{i_{0}}, J\right)>\sum_{i \neq i_{0}} \theta\left(d_{i}, J\right)
$$

If $\left\{x_{0}, \ldots, x_{n-1}\right\}$ is a convex cyclic $n$-gon with side length collection $\left(d_{0}, \ldots, d_{n-1}\right)$ on a hyperbolic circle of radius $J$, then $J \geq \max \left\{d_{i} / 2\right\}$ and, for each $i$, the angle $\theta_{i}$ from $x_{i-1}$ to $x_{i}$ is either $\theta\left(d_{i}, J\right)$ or $2 \pi-\theta\left(d_{i}, J\right)$. If $\theta_{i}=\theta\left(d_{i}, J\right)$ for all $i>0$, then:

$$
d_{0}=\ell^{n}\left(J, d_{1}, \ldots, d_{n-1}\right) \doteq 2 \sinh ^{-1}\left[\sinh J \sin \left(\frac{1}{2} \sum_{i=1}^{n-1} \theta\left(d_{i}, J\right)\right)\right] .
$$

If $\theta_{i}=2 \pi-\theta\left(d_{i_{0}}, J\right)$ for some $i_{0}>0$, then

$$
\sum_{i \neq i_{0}} \theta\left(d_{i}, J\right)<\theta\left(d_{i_{0}}, J\right)
$$

so, in particular, $d_{i_{0}}>d_{i}$ for all $i \neq i_{0}$. In this case,

$$
\begin{aligned}
d_{0} & =\ell^{s}\left(J, d_{1}, \ldots, d_{n-1}\right) \\
& \doteq 2 \sinh ^{-1}\left[\sinh J \sin \left(\frac{1}{2} \theta\left(d_{i_{0}}, J\right)-\frac{1}{2} \sum_{i \neq i_{0}} \theta\left(d_{i}, J\right)\right)\right] .
\end{aligned}
$$

The letters " $n$ " and " $s$ " decorating $\ell$ above stand for "non-separating" and "separating," respectively, and correspond to the left and right sides of Figure 1. On the right, the longest arc separates the others from the center of the circumcircle, whereas no arc on the left has this property. 
Proof. We begin by stipulating some basic facts. First, for any $J>0$ and $\theta_{1}, \ldots, \theta_{n-1} \in(0,2 \pi)$, it is easy to see that any circle $C$ of radius $J$ contains a collection $\left\{x_{0}, \ldots, x_{n-1}\right\}$ such that the angle from $x_{i-1}$ to $x_{i}$ is $\theta_{i}$, for each $i>0$, and that this collection is cyclically ordered if and only if

$$
\sum_{i=1}^{n-1} \theta_{i}<2 \pi .
$$

Second, any such collection is determined up to isometry by the $\theta_{i}$. The orientation preserving isometry group of $\mathbb{H}^{2}$ acts transitively on circles of a fixed radius and includes all rotations around the center of $C$, and for another such collection $\left\{x_{0}^{\prime}, \ldots, x_{n-1}^{\prime}\right\}$ on a circle $C^{\prime}$ of radius $J$, the orientation-preserving isometry taking $C^{\prime}$ to $C$ and $x_{0}^{\prime}$ to $x_{0}$ takes $x_{i}^{\prime}$ to $x_{i}$ for all $i$.

Finally, for any $x$ and $y$ at distance $d>0$ on a circle $C$ of radius $J$, $d \leq 2 J$ by the triangle inequality, and the angle from $x$ to $y$ is $\theta(d, J)$ or $2 \pi-\theta(d, J)$. This follows upon applying Lemma 1.4 to the triangle $T$ spanned by $x, y$ and the center $v$ of $C$. The interior angle of $T$ at $v$ faces the counterclockwise arc from $x$ to $y$ in the former case, and the clockwise arc in the latter.

Now, given $d_{1}, \ldots, d_{n-1} \in(0, \infty)$ and $J \geq \max \left\{d_{i} / 2\right\}$, for any circle $C$ of radius $J$ the relation (1.1) from the proof of Lemma 1.4 implies that a collection $\left\{x_{i}\right\} \subset C$, with the property that the angle $\theta_{i}$ from $x_{i-1}$ to $x_{i}$ is $\theta\left(d_{i}, J\right)$ for each $i>0$, has $\operatorname{dist}\left(x_{i-1}, x_{i}\right)=d_{i}$ for each $i>0$. This still holds if $\theta_{i_{0}}=2 \pi-\theta\left(d_{i_{0}}, J\right)$ for some $i_{0}$ since, in this case, the interior angle of the triangle determined by $x_{i-1}, x_{i}$ and the center of $C$ faces the clockwise arc from $x_{i_{0}-1}$ to $x_{i_{0}}$ and hence still equals $\theta\left(d_{i_{0}}, J\right)$. Note also in this case that

$$
\sum \theta_{i}<2 \pi,
$$

if and only if,

$$
\theta\left(d_{i_{0}}, J\right)>\sum_{i \neq i_{0}} \theta\left(d_{i}, J\right) .
$$

Now suppose $\left\{x_{0}, \ldots, x_{n-1}\right\}$ is a convex cyclic $n$-gon with side length collection $\left(d_{0}, \ldots, d_{n-1}\right)$ on a hyperbolic circle $C$ of radius $J$ and, for each $i>0$, let $\theta_{i}$ be the angle from $x_{i-1}$ to $x_{i}$. From above we have 
$\theta_{i}=\theta\left(d_{i}, J\right)$ or $\theta_{i}=2 \pi-\theta\left(d_{i}, J\right)$ for each $i>0$, and

$$
\sum \theta_{i}<2 \pi
$$

Since $\theta\left(d_{i}, J\right) \in(0, \pi], \theta_{i}$ can be $2 \pi-\theta\left(d_{i}, J\right)$ for at most one $i>0$. Moreover, if $i_{0}$ is such an index, then

$$
\sum_{i \neq i_{0}} \theta\left(d_{i}, J\right)<\theta\left(d_{i_{0}}, J\right)
$$

as above. In particular, $d_{i_{0}}>d_{i}$ for each $i \neq i_{0}$ since one easily sees that $\theta(d, J)$ increases with $d$ for fixed $J$.

Below, let us say that $\left\{x_{0}, \ldots, x_{n-1}\right\}$ satisfies case $n$ if $\theta_{i}<\pi$ for each $i$, and case $s$ otherwise. In case $n$, the angle from $x_{n-1}$ to $x_{0}$ is

$$
2 \pi-\sum_{i=1}^{n-1} \theta\left(d_{i}, J\right)
$$

whereas, in case $s$, this angle is

$$
\theta\left(d_{i_{0}}, J\right)-\sum_{i \neq i_{0}} \theta\left(d_{i}, J\right)
$$

Appealing to Lemma 1.4 translates this angle measure into the functions $\ell^{n}$ and $\ell^{s}$ recorded above (in cases $n$ and $s$, respectively).

Note that, in case $n$, the triangle determined by $x_{0}, x_{n-1}$ and the circle center $v$ has interior angle

$$
\theta=2 \pi-\sum_{i=1}^{n-1} \theta\left(d_{i}, J\right)
$$

at $v$ if $\theta \leq \pi$, but the interior angle here is $2 \pi-\theta$ if $\theta>\pi$. But this makes no difference in $\ell^{n} \operatorname{since} \sin x=\sin (\pi-x)$.

Proposition 1.6. The functions $\ell^{n}$ and $\ell^{s}$ from Proposition 1.5 are continuous on their domain:

$$
\left\{\left(J, d_{1}, \ldots, d_{n-1}\right) \mid n \geq 3, d_{i}>0 \text { for all } i, \text { and } J \geq \max \left\{d_{i} / 2\right\}_{i=1}^{n-1}\right\} \text {. }
$$

They are smooth on its interior, with identical values on its frontier:

$$
\left\{\left(J, d_{1}, \ldots, d_{n-1}\right) \mid J=\max \left\{d_{i} / 2\right\}_{i=1}^{n-1}\right\} .
$$


Now fix $\left(d_{1}, \ldots, d_{n-1}\right) \in\left(\mathbb{R}^{+}\right)^{n-1}$ for $n \geq 3$, let $D=\max \left\{d_{i}\right\}_{i=1}^{n-1}$ and, restricting $\ell^{n}$ and $\ell^{s}$ to $[D / 2, \infty) \times\left\{\left(d_{1}, \ldots, d_{n-1}\right)\right\}$, take them as functions of $J$. Then

$$
\frac{\partial \ell^{n}}{\partial J}(J)>0 \quad \text { for all } J>J_{0},
$$

defined by:

$$
J_{0}=\min \left\{J \geq D / 2 \mid \sum_{i=1}^{n-1} \theta\left(d_{i}, J\right) \leq 2 \pi\right\} .
$$

Moreover,

$$
\lim _{J \rightarrow \infty} \ell^{n}(J)=2 \sinh ^{-1}\left(\sum_{i=1}^{n-1} \sinh \left(d_{i} / 2\right)\right) .
$$

For $i_{0} \in\{0, \ldots, n-1\}$, there exists $J \geq D / 2$ such that

$$
\theta\left(d_{i_{0}}, J\right) \geq \sum_{i \neq i_{0}} \theta\left(d_{i}, J\right)
$$

if and only if $d_{i_{0}}=D$ and

$$
\sum_{i=1}^{n-1} \theta\left(d_{i}, D / 2\right) \leq 2 \pi .
$$

In particular, $J_{0}=D / 2$. If this is the case, then

$$
\left\{J \geq \frac{D}{2} \mid \theta\left(d_{i_{0}}, J\right) \geq \sum_{i \neq i_{0}} \theta\left(d_{i}, J\right)\right\}
$$

is an interval, $I$, and

$$
\frac{\partial \ell^{s}}{\partial J}(J)<0
$$

for $J$ in its interior.

If

$$
\sinh \left(\frac{d_{i_{0}}}{2}\right) \geq \sum_{i \neq i_{0}} \sinh \left(\frac{d_{i}}{2}\right)
$$


then

$$
\sum_{i=1}^{n-1} \theta\left(d_{i}, \frac{D}{2}\right) \leq 2 \pi
$$

$I$, as defined above, is $\left[d_{i_{0}} / 2, \infty\right)$, and

$$
\lim _{J \rightarrow \infty} \ell^{s}(J)=2 \sinh ^{-1}\left(\sinh \left(\frac{d_{i_{0}}}{2}\right)-\sum_{i \neq i_{0}} \sinh \left(\frac{d_{i}}{2}\right)\right) .
$$

If $\sinh \left(d_{i_{0}} / 2\right)<\sum_{i \neq i_{0}} \sinh \left(d_{i} / 2\right)$, but $\sum_{i=1}^{n-1} \theta\left(d_{i}, D / 2\right) \leq 2 \pi$, then $I=\left[d_{i_{0}} / 2, J_{1}\right]$ for some $J_{1}<\infty$, and $\ell^{s}\left(J_{1}\right)=0$.

Remark 1.7. Below, when a value of $\left(d_{1}, \ldots, d_{n-1}\right)$ is clear from the context, we will frequently abbreviate $\ell^{n}\left(J, d_{1}, \ldots, d_{n-1}\right)$ to $\ell^{n}(J)$ without comment, and likewise for $\ell^{s}$.

Proof. The continuity and smoothness properties of $\ell^{n}$ and $\ell^{s}$, and the description of their domain, follow directly from those of the functions involved in their definition. In particular, see Lemma 1.4 for those of $\theta(d, J)$. That $\ell^{n}(J)=\ell^{s}(J)$ if $J=\max \left\{d_{i} / 2\right\}$ follows from the fact that $\theta(d, d / 2)=\pi$ and $\sin (\pi / 2-x)=\sin (\pi / 2+x)$ for any $x$.

We now fix $\left(d_{1}, \ldots, d_{n-1}\right) \in\left(\mathbb{R}^{+}\right)^{n-1}$, let $D=\max \left\{d_{i}\right\}$ and, taking the restriction of $\ell^{n}$ to $[D / 2, \infty) \times\left\{\left(d_{1}, \ldots, d_{n-1}\right\}\right.$ as a function of $J$, prove its properties described in the statement. We first use Lemma 1.4 to compute its derivatives. Take

$$
\theta_{0}(J)=\sum_{i=1}^{n-1} \theta\left(d_{i}, J\right)
$$

below. Then $\partial \ell^{n} / \partial J$ is:

$$
\frac{2 \cosh J}{\cosh \left(\ell^{n}(J) / 2\right)}\left[\sin \left(\frac{\theta_{0}(J)}{2}\right)-\cos \left(\frac{\theta_{0}(J)}{2}\right) \sum_{i=1}^{n-1} \tan \left(\frac{\theta\left(d_{i}, J\right)}{2}\right)\right] .
$$


Subclaim 1.8. For

$$
\begin{gathered}
J>J_{0}=\min \left\{J \geq \max \left\{\frac{d_{i}}{2}\right\} \mid \sum_{i=1}^{n-1} \theta\left(d_{i}, J\right) \leq 2 \pi\right\}, \\
\frac{\partial}{\partial J} \ell^{n}(J)>0 .
\end{gathered}
$$

If $\theta_{0}\left(J_{0}\right)<\pi$, let $J_{1}=J_{0}$; otherwise, define $J_{1}>J_{0}$ by the equation $\theta_{0}\left(J_{1}\right)=\pi$. If $J_{1}>J_{0}$, then for $J_{0}<J \leq J_{1}$, the derivative (1.2) is positive since $\cos \left(\theta_{0}(J) / 2\right) \leq 0$ for such $\bar{J}$. On $\left(J_{1}, \infty\right)$ we use the following claim.

Claim 1.9. If

$$
0<\sum_{i=1}^{n} \alpha_{i}<\pi / 2
$$

then

$$
\tan \left(\sum_{i=1}^{n} \alpha_{i}\right)>\sum_{i=1}^{n} \tan \left(\alpha_{i}\right)
$$

Proof of Claim 1.9. For $0 \leq \alpha \leq \beta$ with $\alpha+\beta<\pi / 2$, we have:

$$
\tan (\alpha+\beta)=\frac{\tan \alpha+\tan \beta}{1-\tan \alpha \tan \beta},
$$

a standard angle-addition identity. Since $\alpha<\pi / 2-\beta$ and sine is increasing on $(0, \pi / 2)$ we have $\sin \alpha<\sin (\pi / 2-\beta)=\cos \beta$. Similarly, $\cos \alpha>\sin \beta$, and so $0<\tan \alpha \tan \beta<1$. Hence, $\tan \alpha+\tan \beta<$ $\tan (\alpha+\beta)$, and the claim follows by induction.

The claim implies that

$$
\sum_{i=1}^{n-1} \tan \left(\frac{\theta\left(d_{i}, J\right)}{2}\right)<\tan \left(\frac{\theta_{0}(J)}{2}\right)
$$

hence, $\partial / \partial J \ell^{n}(J)>0$ on $\left(J_{1}, \infty\right)$. This proves Subclaim 1.8. We next show: 


\section{Subclaim 1.10.}

$$
\lim _{J \rightarrow \infty} \ell^{n}(J)=2 \sinh ^{-1}\left(\sum_{i=1}^{n-1} \sinh \left(\frac{d_{i}}{2}\right)\right) .
$$

Again, let

$$
\theta_{0}(J)=\sum_{i=1}^{n-1} \theta\left(d_{i}, J\right)
$$

The limit of $\sinh J \sin \left(\theta_{0}(J) / 2\right)$ is of the form $\infty \cdot 0$ by Lemma 1.4 , so replacing $\sinh J$ by $1 /(1 / \sinh J)$ and applying l'Hôpital's rule gives:

$$
\lim _{J \rightarrow \infty} \sinh J \sin \left(\theta_{0}(J) / 2\right)=\lim _{J \rightarrow \infty} \cos \left(\theta_{0}(J) / 2\right) \sum_{i=1}^{n-1} \frac{\sinh J \sinh (d / 2)}{\sqrt{\sinh ^{2} J-\sinh ^{2}(d / 2)}} .
$$

Here we have used a formula from Lemma 1.4. Evaluating the limit gives Subclaim 1.10.

We now turn our attention to $\ell^{s}$.

Claim 1.11. For positive real numbers $d_{1}, \ldots, d_{n-1}, n \geq 3$, and any fixed $i_{0}$,

$$
\lim _{J \rightarrow \infty} \frac{\sum_{i \neq i_{0}} \theta\left(d_{i}, J\right)}{\theta\left(d_{i_{0}}, J\right)}=\lim _{J \rightarrow \infty} \frac{\sum_{i \neq i_{0}}(\partial / \partial J) \theta\left(d_{i}, J\right)}{(\partial / \partial J) \theta\left(d_{i_{0}}, J\right)}=\frac{\sum_{i \neq i_{0}} \sinh \left(d_{i} / 2\right)}{\sinh \left(d_{i_{0}} / 2\right)} .
$$

Moreover, if $d_{i_{0}}>d_{i}$ for all $i \neq i_{0}$, then

$$
\frac{\sum_{i \neq i_{0}}(\partial / \partial J) \theta\left(d_{i}, J\right)}{(\partial / \partial J) \theta\left(d_{i_{0}}, J\right)}
$$

is strictly increasing, and it is less than 1 for every $J>d_{i_{0}} / 2$, such that $\theta\left(d_{i_{0}}, J\right) \geq \sum_{i \neq i_{0}} \theta\left(d_{i}, J\right)$.

Proof of Claim 1.11. Since each $\theta\left(d_{i}, J\right) \rightarrow 0$ as $J \rightarrow \infty$, the lefthand equation above follows from l'Hôpital's rule. Directly substituting the formulas of Lemma 1.4 gives:

$$
\frac{\sum_{i \neq i_{0}}(\partial / \partial J) \theta\left(d_{i}, J\right)}{(\partial / \partial J) \theta\left(d_{i_{0}}, J\right)}=\sum_{i \neq i_{0}} \frac{\sinh \left(d_{i} / 2\right)}{\sinh \left(d_{i_{0}} / 2\right)} \sqrt{\frac{\sinh ^{2} J-\sinh ^{2}\left(d_{i_{0}} / 2\right)}{\sinh ^{2} J-\sinh ^{2}\left(d_{i} / 2\right)}}
$$


One can easily verify the limit computation. Moreover, a slight rearrangement gives:

$$
\frac{\sum_{i \neq i_{0}}(\partial / \partial J) \theta\left(d_{i}, J\right)}{(\partial / \partial J) \theta\left(d_{i_{0}}, J\right)}=\sum_{i \neq i_{0}} \frac{\sinh \left(d_{i} / 2\right)}{\sinh \left(d_{i_{0}} / 2\right)} \sqrt{1-\frac{\sinh ^{2}\left(d_{i_{0}} / 2\right)-\sinh ^{2}\left(d_{i} / 2\right)}{\sinh ^{2} J-\sinh ^{2}\left(d_{i} / 2\right)}} .
$$

This makes it clear that the derivative ratio is increasing. To prove the final assertion we rewrite it using the tangent formula of Lemma 1.4 and apply Claim 1.9.

Subclaim 1.12. If $\sinh \left(d_{i_{0}} / 2\right) \geq \sum_{i \neq i_{0}} \sinh \left(d_{i} / 2\right)$, then $\theta\left(d_{i_{0}}, J\right)>$ $\sum_{i \neq i_{0}} \theta\left(d_{i}, J\right)$, for all $J \geq d_{i_{0}} / 2$, and

$$
\lim _{J \rightarrow \infty} \ell^{s}(J)=2 \sinh ^{-1}\left(\sinh \left(d_{i_{0}} / 2\right)-\sum_{i \neq i_{0}} \sinh \left(d_{i} / 2\right)\right) .
$$

If $\sinh \left(d_{i_{0}} / 2\right) \geq \sum_{i \neq i_{0}} \sinh \left(d_{i} / 2\right)$, then by Claim 1.11, the derivative ratio

$$
\frac{\sum_{i \neq i_{0}}(\partial / \partial J) \theta\left(d_{i}, J\right)}{(\partial / \partial J) \theta\left(d_{i_{0}}, J\right)},
$$

is less than 1 for all $J>d_{i_{0}} / 2$, so $\theta\left(d_{i_{0}}, J\right)$ decreases more quickly than $\sum_{i \neq i_{0}} \theta\left(d_{i}, J\right)$ on the entire interval $\left(d_{i_{0}} / 2, \infty\right)$. It follows that, if $\theta\left(d_{i_{0}}, J\right) \leq \sum_{i \neq i_{0}} \theta\left(d_{i}, J\right)$, for any $J \geq d_{i_{0}} / 2$, then for any $\epsilon>0$, there exists a $\delta>0$ such that

$$
\theta\left(d_{i_{0}}, J\right)<\sum_{i \neq i_{0}} \theta\left(d_{i}, J\right)-\delta \text { on }[J+\epsilon, \infty) .
$$

But, this would violate the fact that both of these functions limit to 0 (by Lemma 1.4), a contradiction.

The limit computation is an application of l'Hôpital's rule analogous to Subclaim 1.10.

Subclaim 1.13. If $\theta\left(d_{i_{0}}, J\right) \geq \sum_{i \neq i_{0}} \theta\left(d_{i}, J\right)$, for some $J \geq d_{i_{0}} / 2$, then the set $I$ of all $J$ for which this inequality holds is an interval with left endpoint $d_{i_{0}} / 2$. If

$$
\sinh \left(d_{i_{0}} / 2\right)<\sum_{i \neq i_{0}} \sinh \left(d_{i} / 2\right),
$$


then $I=\left[d_{i_{0}} 2, J_{1}\right]$ is compact, with $\ell^{s}\left(J_{1}\right)=0$.

These assertions follow from Claim 1.11. The final assertion implies that $I$ is "open to the left" on $\left[d_{i_{0}} / 2, \infty\right)$ so, since it is also closed, it is of the form $\left[d_{i_{0}} / 2, \infty\right)$ or $\left[d_{i_{0}} / 2, J_{1}\right]$, for some $J_{1}<\infty$. But, if $\sinh \left(d_{i_{0}} / 2\right)<\sum_{i \neq i_{0}} \sinh \left(d_{i} / 2\right)$, then the limit computation of Claim 1.11 implies

$$
\lim _{J \rightarrow \infty} \frac{\sum_{i \neq i_{0}} \theta\left(d_{i}, J\right)}{\theta\left(d_{i_{0}}, J\right)}>1,
$$

so $I$ must be of the latter form. In this case, $\ell^{s}\left(J_{1}\right)=0$ by continuity since $\ell^{s}(J)<0$ for all $J>J_{1}$.

It remains to show that $\partial \ell^{s} / \partial J<0$ in the interior of $I$. Here, let

$$
\theta_{0}(J)=\theta\left(d_{i_{0}}, J\right)-\sum_{i \neq i_{0}} \theta\left(d_{i}, J\right)
$$

Then $(\partial / \partial J) \ell^{s}(J)$ has the following description:

$$
\begin{array}{r}
\frac{2 \cosh J}{\cosh \left(\ell^{s}(J) / 2\right)}\left[\sin \left(\theta_{0}(J) / 2\right)-\cos \left(\theta_{0}(J) / 2\right)\right. \\
\left.\cdot\left(\tan \left(\theta\left(d_{i_{0}}, J\right) / 2\right)-\sum_{i \neq i_{0}} \tan \left(\theta\left(d_{i}, J\right) / 2\right)\right)\right] .
\end{array}
$$

One shows that

$$
\tan \left(\theta\left(d_{i_{0}}, J\right) / 2\right)-\sum_{i \neq i_{0}} \tan \left(\theta\left(d_{i}, J\right) / 2\right)>\tan \left(\theta_{0} / 2\right)
$$

in two steps, first applying Claim 1.9 to

$$
\sum_{i \neq i_{0}} \tan \left(\theta\left(d_{i}, J\right) / 2\right),
$$

then using the inequality $\tan (\alpha-\beta)<\tan \alpha-\tan \beta$ (which follows from the angle-addition identity for tangent). This shows that

$$
\frac{\partial}{\partial J} \ell^{s}(J)<0
$$

Proposition 1.14. For positive real numbers $d_{0}, \ldots, d_{n-1}, n \geq 3$, there is a cyclic $n$-gon with side length collection $\left(d_{0}, \ldots, d_{n-1}\right)$ if 
and only if, for each $i, \sinh \left(d_{i} / 2\right)<\sum_{j \neq i} \sinh \left(d_{j} / 2\right)$. Two cyclic $n$-gons, with side length collections $\left(d_{0}, \ldots, d_{n-1}\right)$ and $\left(d_{0}^{\prime}, \ldots, d_{n-1}^{\prime}\right)$, are isometric if and only if, $d_{i}^{\prime}=d_{\sigma(i)}$ for a cyclic permutation $\sigma$. In particular, circumcircle radius is uniquely determined by $\left(d_{0}, \ldots, d_{n-1}\right)$. Moreover, it is symmetric as a function of $\left(d_{0}, \ldots, d_{n-1}\right)$.

Proof. We first prove existence. For a fixed collection $d_{0}, \ldots, d_{n-1}$, $n \geq 3$, of positive real numbers such that $\sinh \left(d_{i} / 2\right)<\sum_{j \neq i} \sinh \left(d_{j} / 2\right)$, for each $i$, we fix $i_{0}>0$ such that $d_{i_{0}}$ is maximal among $d_{1}, \ldots, d_{n-1}$, and consider the following three cases.

$$
\sum_{i=1}^{n-1} \theta\left(d_{i}, d_{i_{0}} / 2\right)>2 \pi,
$$

$$
\sum_{i=1}^{n-1} \theta\left(d_{i}, d_{i_{0}} / 2\right) \leq 2 \pi,
$$

but $\sinh \left(d_{i_{0}} / 2\right)<\sum_{i=1, i \neq i_{0}}^{n-1} \sinh \left(d_{i} / 2\right)$,

$$
\sinh \left(d_{i_{0}} / 2\right) \geq \sum_{\substack{i=1 \\ i \neq i_{0}}}^{n-1} \sinh \left(d_{i} / 2\right) ;
$$

hence, $\sum_{i=1}^{n-1} \theta\left(d_{i}, d_{i_{0}} / 2\right) \leq 2 \pi$, by Proposition 1.6.

If $d_{1}, \ldots, d_{n-1}$ satisfies (i), then for $J_{0}$ as in Proposition 1.6, $\ell^{n}\left(J_{0}\right)=0$. Since $\ell^{n}$ limits to $2 \sinh ^{-1}\left(\sum_{i=1}^{n-1} \sinh \left(d_{i} / 2\right)\right)$, and, since $\sinh \left(d_{0} / 2\right)<\sum_{i=1}^{n-1} \sinh \left(d_{i} / 2\right)$, there exists $J>J_{0}$ such that $d_{0}=$ $\ell^{n}(J)$. Since $J>J_{0}, \sum_{i=1}^{n-1} \theta\left(d_{i}, J\right)<2 \pi$. For this $J$, Proposition 1.5 thus implies that a collection, $x_{0}, \ldots, x_{n-1}$, arranged on a circle of radius $J$, such that the angle from $x_{i-1}$ to $x_{i}$ is $\theta\left(d_{i}, J\right)$ for each $i>0$ is a cyclic $n$-gon with side length collection $\left(d_{0}, \ldots, d_{n-1}\right)$.

In case (ii), let $D_{0}=\ell^{n}\left(d_{i_{0}} / 2\right)=\ell^{s}\left(d_{i_{0}} / 2\right)$. Proposition 1.6 implies that the range of $\ell^{n}$ is $\left[D_{0}, 2 \sinh ^{-1}\left(\sum_{i=1}^{n-1} \sinh \left(d_{i} / 2\right)\right)\right)$, and that of $\ell^{s}$ is $\left[0, D_{0}\right]$, so as in case (i) there exists $J \geq d_{i_{0}} / 2$ such that either $d_{0}=\ell^{n}(J)$ or $\ell^{s}(J)$. In the former instance, we arrange $x_{0}, \ldots, x_{n-1}$, so that the angle from $x_{i}$ to $x_{i-1}$ is $\theta\left(d_{i}, J\right)$ for all $i$; and, in the latter, so that this holds for all $i$ but $i_{0}$, with the angle from $x_{i_{0}-1}$ to $x_{i_{0}}$ equal 
to $2 \pi-\theta\left(d_{i_{0}}, J\right)$. Proposition 1.5 again implies that $x_{0}, \ldots, x_{n-1}$, is a cyclic $n$-gon with the requisite side lengths.

Case (iii) parallels case (ii). We must only note in addition that, rearranging the inequality $\sinh \left(d_{i_{0}} / 2\right)<\sum_{j \neq i_{0}} \sinh \left(d_{j} / 2\right)$, for $j$ between 0 and $n-1$, gives

$$
\sinh \left(d_{0} / 2\right)>\sinh \left(d_{i_{0}} / 2\right)-\sum_{i \neq i_{0}} \sinh \left(d_{i} / 2\right),
$$

for $i$ between 1 and $n-1$. This proves existence of a cyclic $n$-gon with side length collection, $d_{0}, \ldots, d_{n-1}$, provided that $\sinh \left(d_{i} / 2\right)<$ $\sum_{j \neq i} \sinh \left(d_{j} / 2\right)$, for each $i$.

If there is a cyclic $n$-gon with side length collection $d_{0}, \ldots, d_{n-1}$, then by Proposition 1.5, either $d_{0}=\ell^{n}(J)$ or $d_{0}=\ell^{s}(J)$ for $\ell^{n}$ and $\ell^{s}$, as defined there, where $J$ is the circumcircle radius. The range of $\ell^{n}$ has supremum

$$
2 \sinh ^{-1}\left(\sum_{i=1}^{n-1} \sinh \left(d_{i} / 2\right)\right),
$$

which is not attained, and if $\ell^{s}$ is defined, it decreases from the minimum $D_{0}$, defined above, of $\ell^{n}$. It follows that

$$
\sinh \left(d_{0} / 2\right)<\sum_{i=1}^{n-1} \sinh \left(d_{i} / 2\right)
$$

Moreover, if $d_{i_{0}}$ is maximal among $d_{1}, \ldots, d_{n-1}$, we claim that

$$
\sinh \left(d_{0} / 2\right)>\sinh \left(d_{i_{0}} / 2\right)-\sum_{i \neq i_{0}} \sinh \left(d_{i_{0}} / 2\right) .
$$

If $d_{1}, \ldots, d_{n-1}$ satisfies case (iii) above, this holds because the range of $\ell^{s}$ is bounded below by

$$
2 \sinh ^{-1}\left(\sinh \left(d_{i_{0}} / 2\right)-\sum_{i \neq i_{0}} \sinh \left(d_{i} / 2\right)\right),
$$

by Proposition 1.6, and that of $\ell^{n}$ by $D_{0}$. In case (ii), the right-hand side of the claimed inequality is less than 0 by hypothesis. This also holds in case (i) since, by Proposition 1.6, $\sum_{i=1}^{n-1} \theta\left(d_{i}, d_{i_{0}} / 2\right) \leq 2 \pi$, if $\sinh \left(d_{i_{0}} / 2\right) \geq \sum_{i \neq i_{0}} \sinh \left(d_{i} / 2\right)$. The claim is thus proved, and it follows that $\sinh \left(d_{i} / 2\right)<\sum_{j \neq i} \sinh \left(d_{j} / 2\right)$, for all $i$. 
For a given $d_{0}, \ldots, d_{n-1}$ with $\sinh \left(d_{i} / 2\right) \leq \sum_{j \neq i} \sinh \left(d_{j} / 2\right)$, for all $i$, we claim that there is only one $J$ such that $d_{0}=\ell^{n}(J)$ or $\ell^{s}(J)$, i.e., that the circumcircle radius is uniquely determined by the side length collection. To this end, we note first that cases (i), (ii) and (iii) above are mutually exclusive. Moreover, $\ell^{n}$ and $\ell^{s}$ are each strictly monotone, and their ranges intersect only at the single point,

$$
D_{0}=\ell^{n}\left(d_{i_{0}} / 2\right)=\ell^{s}\left(d_{i_{0}} / 2\right) \text {. }
$$

The claim follows.

It is further evident by inspecting the definitions of $\ell^{n}$ and $\ell^{s}$ that they are symmetric in $d_{1}, \ldots, d_{n-1}$, so the circumcircle radius $J$ determined by $d_{0}, \ldots, d_{n-1}$ is invariant under any reordering of the final $n-1$ side lengths. We claim it is also invariant under cyclic permutations of $\left(d_{0}, \ldots, d_{n-1}\right)$.

The key here is that Proposition 1.6 also applies to the restrictions of $\ell^{n}$ and $\ell^{s}$ to $\left[D_{1} / 2, \infty\right)\left\{\left(d_{2}, \ldots, d_{n-1}, d_{0}\right)\right\}$, where $D_{1}=\max \left\{d_{i}\right\}_{i \neq 1}$. The arguments above thus imply that

$$
d_{1}=\ell^{n}\left(J_{1}, d_{2}, \ldots, d_{n-1}, d_{0}\right) \quad \text { or } \quad d_{1}=\ell^{s}\left(J_{1}, d_{2}, \ldots, d_{n-1}, d_{0}\right),
$$

for a unique $J_{1} \in\left[D_{1} / 2, \infty\right) . J_{1}=J$ follows from uniqueness with a little case-matching.

If $d_{0}=\ell^{n}\left(J, d_{1}, \ldots, d_{n-1}\right)$, then, plugging into the definition of $\theta(d, J)$ from Lemma 1.4, gives:

$$
\theta\left(d_{0}, J\right)=\sum_{i=1}^{n-1} \theta\left(d_{i}, J\right) \quad \text { or } \quad \theta\left(d_{0}, J\right)=2 \pi-\sum_{i=1}^{n-1} \theta\left(d_{i}, J\right) .
$$

The ambiguity arises because $\sin ^{-1}$ takes values in $[-\pi / 2, \pi / 2]$. For $x \in[0, \pi], \sin ^{-1}(\sin x)=x$ if $x \leq \pi / 2$ and $\pi-x$ otherwise. Thus, $\sum_{i=1}^{n-1} \theta\left(d_{i}, J\right)$ is at most $\pi$ in the former case and at least $\pi$ in the latter. In the former, rearranging gives:

$$
\theta\left(d_{1}, J\right)=\theta\left(d_{0}, J\right)-\sum_{i=2}^{n-1} \theta\left(d_{i}, J\right) \in(0, \pi)
$$

Therefore, $d_{1}=\ell^{s}\left(J, d_{2}, \ldots, d_{n-1}, d_{0}\right)$ in this case. In the latter case, we have $\theta\left(d_{1}, J\right)=2 \pi-\sum_{i \neq 1} \theta\left(d_{i}, J\right)$, so $d_{1}=\ell^{n}\left(J, d_{2}, \ldots, d_{n-1}, d_{0}\right)$. 
Here we use $\sin (\pi-x)=\sin x$. In either case, we conclude from uniqueness that $J=J_{1}$.

If $d_{0}=\ell^{s}\left(J, d_{1}, \ldots, d_{n-1}\right)$, then for $d_{i_{0}}$ maximal among $d_{1}, \ldots, d_{n-1}$ :

$$
\theta\left(d_{0}, J\right)=\theta\left(d_{i_{0}}, J\right)-\sum_{\substack{i>0 \\ i \neq i_{0}}} \theta\left(d_{i}, J\right) .
$$

There are two cases, handled similarly to the above. If $i_{0}=1$, then $d_{1}=\ell^{n}\left(J, d_{2}, \ldots, d_{n-1}, d_{0}\right)$, otherwise $d_{1}=\ell^{s}\left(J, d_{2}, \ldots, d_{n-1}, d_{0}\right)$. In each case, we again conclude $J=J_{1}$, which proves the claim and also shows that $J=J\left(d_{0}, \ldots, d_{n-1}\right)$ is symmetric.

We finally show that cyclic $n$-gons with side length collections $\left(d_{0}, \ldots, d_{n-1}\right)$ and $\left(d_{0}^{\prime}, \ldots, d_{n-1}^{\prime}\right)$ are isometric if and only if $d_{i}^{\prime}=d_{\sigma(i)}$, for each $i$, where $\sigma$ is a fixed cyclic permutation. The "only if" direction is in Remark 1.3, so let us suppose for cyclic $n$-gons $\left\{x_{i}\right\}$ and $\left\{x_{i}^{\prime}\right\}$ with respective side length collections above that there is a cyclic permutation $\sigma$ so that $d_{i}^{\prime}=d_{\sigma(i)}$ for all $i$. Upon cyclically reordering the $x_{i}$ we may assume that $d_{i}^{\prime}=d_{i}$ for each $i$ (again see Remark 1.3). We have already shown that $\left\{x_{i}\right\}$ and $\left\{x_{i}^{\prime}\right\}$ have the same circumcircle radius $J$, and moreover, that $d_{0}$ is only one of $\ell^{n}(J)$ or $\ell^{s}(J)$. Proposition 1.5 now implies that $\left\{x_{i}\right\}$ and $\left\{x_{i}^{\prime}\right\}$ are isometric.

Having established existence and uniqueness of cyclic polygons by carefully analyzing the functions $\ell^{n}$ and $\ell^{s}$, we will use the following lemma to avoid further consideration of $\ell^{s}$.

Lemma 1.15. Suppose, for $n \geq 3$, that a cyclic $n$-gon with side length collection $\left(d_{0}, \ldots, d_{n-1}\right)$ and circumcircle radius $J$ has $d_{0}=$ $\max \left\{d_{i}\right\}_{i=0}^{n-1}$. Then the following hold: $d_{0}=\ell^{n}\left(J, d_{1}, \ldots, d_{n-1}\right)$, for $\ell^{n}$ as defined in Proposition 1.5; and $J>D / 2$, for $D=\max \left\{d_{i}\right\}_{i=1}^{n-1}$. Furthermore, both assertions hold for all side length collections of cyclic $n$-gons near $\left(d_{0}, \ldots, d_{n-1}\right)$ in $\mathbb{R}^{n}$.

Proof. By Proposition 1.5, either $d_{0}=\ell^{n}\left(J, d_{1}, \ldots, d_{n-1}\right)$ or $d_{0}=$ $\ell^{s}\left(J, d_{1}, \ldots, d_{n-1}\right)$ as defined there. But $\ell^{s}$ is decreasing in $J$ and defined only for $J \geq D / 2$ by Proposition 1.6 , where $D=\max \left\{d_{i}\right\}_{i=1}^{n-1} \leq$ 
$d_{0}$ by hypothesis. We calculate:

$\ell^{s}\left(D / 2, d_{1}, \ldots, d_{n-1}\right)<2 \sinh ^{-1}\left[\sinh (D / 2) \sin \left(\frac{1}{2} \theta(D, D / 2)\right)\right]=D$.

Since $\ell^{s}$ is continuous, this inequality holds on a neighborhood of $\left(d_{1}, \ldots, d_{n-1}\right)$ in $\mathbb{R}^{n}$, and it follows that there is a neighborhood of $\left(d_{0}, \ldots, d_{n-1}\right)$ such that $d_{0}=\ell^{n}(J)$ for each side length collection in this neighborhood.

By Proposition 1.6, we have $\ell^{n}\left(D / 2, d_{1}, \ldots, d_{n-1}\right)=\ell^{s}\left(D / 2, d_{1}, \ldots\right.$, $\left.d_{n-1}\right)<D$, so, since $\ell^{n}$ is continuous, this inequality also holds on an entire neighborhood of $\left(d_{0}, \ldots, d_{n-1}\right)$. It implies that $J>D / 2$ here.

1.2. Parametrizing cyclic polygons. Having proved the existence and uniqueness of cyclic hyperbolic $n$-gons, in this section, we will first parametrize them by side length using an open set in $\left(\mathbb{R}^{+}\right)^{n}$. Then we will describe some subspaces of this set corresponding to subclasses of cyclic polygons that turn out to be geometrically significant.

Corollary 1.16. For $n \geq 3$, the collection

$\mathcal{A C}_{n}=\left\{\mathbf{d} \mid \sinh \left(d_{i} / 2\right)<\sum_{j \neq i} \sinh \left(d_{j} / 2\right)\right.$ for each $\left.i \in\{0, \ldots, n-1\}\right\}$,

(taking $\left.\mathbf{d}=\left(d_{0}, \ldots, d_{n-1}\right) \in\left(\mathbb{R}^{+}\right)^{n}\right)$ parametrizes isometry classes of marked cyclic n-gons in $\mathbb{H}^{2}$ by their side length collections, where a marking is the choice of a vertex to label $x_{0}$.

This is a direct consequence of Proposition 1.14 since the marking takes care of the cyclic ambiguity of side length collections.

Proposition 1.17. For $n \geq 3$, a cyclic $n$-gon $\left\{x_{0}, \ldots, x_{n-1}\right\}$ is centered if the angle from $x_{i-1}$ to $x_{i}$ is less than $\pi$, for each $i$, or equivalently, if $\sum_{i \neq i_{0}} \theta\left(d_{i}, J\right)>\pi$, where $\left(d_{0}, \ldots, d_{n-1}\right)$ is its side length collection, with $d_{i_{0}}$ maximal, and $J$ is its circumcircle radius. Again, taking $\mathbf{d}=\left(d_{0}, \ldots, d_{n-1}\right) \in\left(\mathbb{R}^{+}\right)^{n}$, the collection,

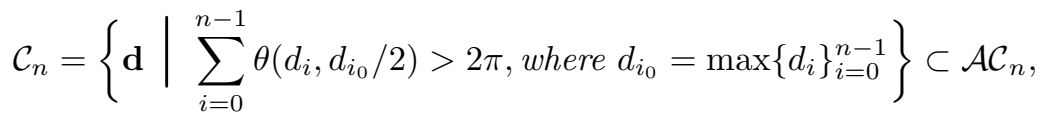


parametrizes marked, centered n-gons in $\mathbb{H}^{2}$ up to hyperbolic isometry. The collection,

$\mathcal{B C}_{n}=\left\{\mathbf{d} \mid \sum_{i=0}^{n-1} \theta\left(d_{i}, d_{i_{0}} / 2\right)=2 \pi\right.$, where $\left.d_{i_{0}}=\max \left\{d_{i}\right\}_{i=0}^{n-1}\right\} \subset \mathcal{A C}_{n}$

parametrizes marked, semicyclic $n$-gons in $\mathbb{H}^{2}$ up to hyperbolic isometry. Here, a cyclic $n$-gon is semicyclic if its circumcircle radius $J$ is $d_{i_{0}} / 2$, or equivalently, if $\sum_{i \neq i_{0}} \theta\left(d_{i}, J\right)=\pi$.

Proof. We first comment on equivalent definitions of centeredness. Recall from Proposition 1.5 that, for each $i$, the angle from $x_{i}$ to $x_{i-1}$ is either $\theta\left(d_{i}, J\right)$, as defined in Lemma 1.4, or $2 \pi-\theta\left(d_{i}, J\right)$. For each $i$, such that this angle is less than $\pi$ it must equal $\theta\left(d_{i}, J\right)$ (recall that $\theta(d, J) \in[0, \pi]$ for all possible $d$ and $J$ ). If this holds for all $i$, then since these angles sum to $2 \pi$ it follows in particular that $\sum_{i \neq i_{0}} \theta\left(d_{i}, J\right)=2 \pi-\theta\left(d_{i_{0}}, J\right)>\pi$.

On the other hand, if $\sum_{i \neq i_{0}} \theta\left(d_{i}, J\right)>\pi$, for $i_{0}$ such that $d_{i_{0}}$ is maximal, then the same inequality holds for any other $i_{0}$, since $\theta(d, J)$ increases in $d$ for fixed $J$. It follows that, for each $i$,

$$
2 \pi-\theta\left(d_{i}, J\right)+\sum_{j \neq i} \theta\left(d_{j}, J\right)>2 \pi
$$

so the angle from $x_{i-1}$ to $x_{i}$ is $\theta\left(d_{i}, J\right)<\pi$.

Now suppose a centered or semicyclic $n$-gon has side length collection $\left(d_{0}, \ldots, d_{n-1}\right)$ and circumcircle radius $J$. We may assume that $d_{0}$ is maximal among the $d_{i}$, since circumcircle radius is symmetric in side lengths and the centeredness criterion is invariant under their permutation. Then, by Lemma $1.15, d_{0}=\ell^{n}\left(J, d_{1}, \ldots, d_{n-1}\right)$, so manipulating the definition of $\ell^{n}$ gives:

$$
\sinh \left(d_{0} / 2\right)=\sinh J \sin \left(\frac{1}{2} \sum_{i=1}^{n-1} \theta\left(d_{i}, J\right)\right) \leq \sinh J
$$

It follows that $J \geq d_{0} / 2$ (with equality here and above if and only if the $n$-gon is semicyclic); hence, since $\theta(d, J)$ decreases in $J$,

$$
\sum_{i=0}^{n-1} \theta\left(d_{i}, d_{i_{0}} / 2\right)=\pi+\sum_{i=1}^{n-1} \theta\left(d_{i}, d_{i_{0}} / 2\right) \geq \pi+\sum_{i=1}^{n-1} \theta\left(d_{i}, J\right) \geq 2 \pi .
$$


Again, equality holds if and only if the original $n$-gon is semicyclic, and we find that the side length collection of a centered or semicyclic collection lies in $\mathcal{C}_{n}$ or $\mathcal{B C}_{n}$, respectively.

Now, suppose that $\left(d_{0}, \ldots, d_{n-1}\right) \in \mathcal{C}_{n} \cup \mathcal{B C}_{n}$ has $d_{0}$ maximal. Then, since $\sum_{i=0}^{n-1} \theta\left(d_{i}, d_{0} / 2\right) \geq 2 \pi$, there exists a unique $J \geq d_{0} / 2$ such that $\sum_{i=0}^{n-1} \theta\left(d_{i}, J\right)=2 \pi$, with $J=d_{0} / 2$ if and only if $\sum_{i=0}^{n-1} \theta\left(d_{i}, d_{0} / 2\right)=$ $2 \pi$.

For this $J$, directly substituting $2 \pi-\theta\left(d_{0}, J\right)$ for $\sum_{i=1}^{n-1} \theta\left(d_{i}, J\right)$ in the definition of $\ell^{n}$ and, applying $\theta$, we find that $\theta\left(d_{0}, J\right)=\theta\left(\ell^{n}(J), J\right)$, so $d_{0}=\ell^{n}(J)$. Therefore, $J$ is the circumcircle radius of a cyclic $n$-gon with side length collection $\left(d_{0}, \ldots, d_{n-1}\right)$. If $J=d_{0} / 2$, then the $n$-gon in question is semi-cyclic; otherwise, $\theta\left(d_{0}, J\right)<\pi$, so $\sum_{i=1}^{n-1} \theta\left(d_{i}, J\right)>\pi$, and it is centered.

We note further that, for $\left(d_{0}, \ldots, d_{n-1}\right) \in \mathcal{C}_{n} \cup \mathcal{B C}_{n}$, since $d_{0}=\ell^{n}(J)$ and $\ell^{n}$ increase toward an asymptote of

$$
2 \sinh ^{-1}\left(\sum_{i=1}^{n-1} \sinh \left(d_{i} / 2\right)\right), \quad\left(d_{0}, \ldots, d_{n-1}\right) \in \mathcal{A C}_{n} .
$$

We finally comment on equivalence of the definitions of semicyclicity. For a cyclic $n$-gon with side length collection $\left(d_{0}, \ldots, d_{n-1}\right)$, such that $d_{0}$ is maximal, $d_{0}=\ell^{n}(J)$ by Lemma 1.4. Directly substituting either $J=d_{i_{0}} / 2$ or $\sum_{i=1}^{n-1} \theta\left(d_{i}, J\right)=\pi$ into the formula for $\ell^{n}(J)$ allows one to conclude the other condition.

Proposition 1.18. For each $n \geq 3, \mathcal{B C}_{n}$ is the frontier of $\mathcal{C}_{n}$ in $\mathcal{A C}_{n}$. It is the orbit of

$$
\operatorname{graph}\left(b_{0}\right) \doteq\left\{\left(b_{0}(\mathbf{d}), \mathbf{d}\right) \mid \mathbf{d} \in\left(\mathbb{R}^{+}\right)^{n-1}\right\},
$$

under cyclic permutation of entries, where $b_{0}:\left(\mathbb{R}^{+}\right)^{n-1} \rightarrow \mathbb{R}$ is defined by:

$$
\sum_{i=1}^{n-1} \theta\left(d_{i}, b_{0}(\mathbf{d}) / 2\right)=\pi .
$$

This function is symmetric, smooth and strictly increasing in each variable. It further satisfies $b_{0}\left(d_{1}, \ldots, d_{n-1}\right)>\max \left\{d_{i}\right\}_{i=1}^{n-1}$. In particular, $\mathcal{B C}_{n}$ has $n$ connected components. 
Proof. Proposition 1.17 directly implies that $\mathcal{B C}_{n}$ is the frontier of $\mathcal{C}_{n}$ in $\mathcal{A C}_{n}$, since the quantities involved in the definitions of these sets vary continuously over $\mathcal{A C}_{n}$.

If $\left(d_{0}, \ldots, d_{n-1}\right) \in \mathcal{B C}_{n}$ has maximal entry $d_{i_{0}}$, then, since $\theta\left(d_{i_{0}}, d_{i_{0}} /\right.$ $2)=\pi$, so does $\sum_{i \neq i_{0}} \theta\left(d_{i}, d_{i_{0}} / 2\right)$. For $D=\max \left\{d_{i}\right\}_{i \neq i_{0}}$, we have $\sum_{i \neq i_{0}} \theta\left(d_{i}, D / 2\right)>\pi$, so, since $\theta(d, J)$ strictly decreases in $J$ for each $\left(d_{0}, \ldots, \widehat{d_{i_{0}}}, \ldots, d_{n-1}\right) \in\left(\mathbb{R}^{+}\right)^{n-1}$, the equation $\sum_{i \neq i_{0}} \theta\left(d_{i}, d_{i_{0}} / 2\right)=\pi$ uniquely determines $d_{i_{0}}>D$.

The above implies in particular that $b_{0}:\left(\mathbb{R}^{+}\right)^{n-1} \rightarrow \mathbb{R}$ is uniquely determined by its defining equation, that $b_{0}\left(d_{1}, \ldots, d_{n-1}\right)>\max \left\{d_{i}\right\}$, and that $\mathcal{B C}_{n}$ is the orbit of graph $\left(b_{0}\right)$ under cyclic permutation of entries. Furthermore, since $b_{0}>\max \left\{d_{i}\right\}$, no distinct translates of graph $\left(b_{0}\right)$ under this action intersect each other. We will thus finish the proof by showing that $b_{0}$ is smooth and strictly increasing in each variable.

This is a direct application of the implicit function theorem, which shows that, for each $i$,

$$
\frac{\partial}{\partial d_{i}} b_{0}\left(d_{1}, \ldots, d_{n-1}\right)=-\frac{(\partial \theta / \partial d)\left(d_{i}, b_{0}\left(d_{1}, \ldots, d_{n-1}\right)\right)}{\sum_{i=1}^{n-1}(\partial \theta / \partial J)\left(d_{i}, b_{0}\left(d_{1}, \ldots, d_{n-1}\right)\right)} .
$$

Since $\theta$ strictly increases in $d$ and decreases in $J$, the result follows.

1.3. Smoothness. Having parametrized cyclic $n$-gons by the space $\mathcal{A C}_{n}$, we turn our attention to describing associated geometric quantities as functions on $\mathcal{A C}_{n}$ : circumcircle radius in Proposition 1.20, diagonal lengths in Corollary 1.21, and what turns out to be area in Corollary 1.23. The main result in each case is that the function in question is smooth, although Proposition 1.20 also describes some qualitative features of the circumcircle radius.

Lemma 1.19. For $n \geq 3$, suppose $\mathbf{d}=\left(d_{0}, \ldots, d_{n-1}\right) \in \mathcal{A C}_{n}$ has $d_{0} \geq d_{i}$ for all $i$. The function, $J: \mathcal{A C}_{n} \rightarrow \mathbb{R}^{+}$, that records circumcircle radius is smooth at $\mathbf{d}$, and

$$
\frac{\partial J}{\partial d_{i}}(\mathbf{d})=\frac{1}{2} \frac{ \pm \cosh \left(d_{i} / 2\right) \tanh J \sqrt{\frac{\sinh ^{2} J-\sinh ^{2}\left(d_{0} / 2\right)}{\sinh ^{2} J-\sinh ^{2}\left(d_{i} / 2\right)}}}{\sinh \left(d_{0} / 2\right) \pm \sum_{j=1}^{n-1} \sinh \left(d_{j} / 2\right) \sqrt{\frac{\sinh ^{2} J-\sinh ^{2}\left(d_{0} / 2\right)}{\sinh ^{2} J-\sinh ^{2}\left(d_{j} / 2\right)}}} .
$$


Above, each \pm should be taken as + if $\mathbf{d} \in \mathcal{C}_{n}$ and - otherwise, with one exception: if $\mathbf{d} \in \mathcal{A C}_{n}-\mathcal{C}_{n}$ and $i=0$, the numerator is $\cosh \left(d_{0} / 2\right) \tanh J$.

Proof. Since $d_{0}$ is maximal, Lemma 1.15 gives a neighborhood $U$ of $\mathbf{d}$ in $\mathcal{A C}_{n}$ on which the equation $d_{0}=\ell^{n}\left(J, d_{0}, \ldots, d_{n-1}\right)$ holds everywhere, for $J=J\left(d_{0}, \ldots, d_{n-1}\right)$. We will thus obtain the desired conclusions by applying the implicit function theorem to

$$
F\left(d_{0}, \ldots, d_{n-1}, J\right)=\ell^{n}\left(J, d_{1}, \ldots, d_{n-1}\right)-d_{0} .
$$

Since $J>D / 2$, by Lemma 1.15 , where $D=\max \left\{d_{i}\right\}_{i=1}^{n-1}$, using Lemma 1.4 and inspecting the definition of $\ell^{n}$, we find that $F$ is smooth near $(\mathbf{d}, J)$. Its derivative vector is

$$
\left(-1, \frac{\partial}{\partial d_{1}} \ell^{n}, \ldots, \frac{\partial}{\partial d_{n-1}} \ell^{n}, \frac{\partial}{\partial J} \ell^{n}\right) .
$$

Since $J(\mathbf{d})>J_{0}$, as defined in Proposition 1.6, that result gives $(\partial / \partial J) \ell^{n}(J(\mathbf{d}))>0$. The implicit function theorem thus guarantees that $J$ is smooth at $\mathbf{d}$, and the following relations hold:

$$
\begin{aligned}
& 0=\frac{\partial J}{\partial d_{0}} \frac{\partial \ell^{n}}{\partial J}-1 \\
& 0=\frac{\partial J}{\partial d_{i}} \frac{\partial \ell^{n}}{\partial J}+\frac{\partial \ell^{n}}{\partial d_{i}}, \quad i>0 .
\end{aligned}
$$

We will substitute for $\partial \ell^{n} / \partial J$ in the relations above using (1.2). In that formula,

$$
\theta_{0}(J)=\sum_{i=1}^{n-1} \theta\left(d_{i}, J\right)
$$

and, using the definition of $\ell^{n}$ and the fact that $d_{0}=\ell^{n}(J(\mathbf{d}))$, we obtain:

$$
\begin{aligned}
& \sin \left(\theta_{0}(J) / 2\right)=\frac{\sinh \left(d_{0} / 2\right)}{\sinh J} \\
& \cos \left(\theta_{0}(J) / 2\right)= \pm \frac{\sqrt{\sinh ^{2} J-\sinh ^{2}\left(d_{0} / 2\right)}}{\sinh J} .
\end{aligned}
$$

The \pm above is a - if $\theta_{0}(J)>\pi$ and a + otherwise; by Proposition 1.17, these cases correspond respectively to $\mathbf{d} \in \mathcal{C}_{n}$ and $\mathbf{d} \in \mathcal{A C}_{n}-\mathcal{C}_{n}$. We 
further substitute

$$
\frac{\sinh \left(d_{i} / 2\right)}{\sqrt{\sinh ^{2} J-\sinh ^{2}\left(d_{i} / 2\right)}}
$$

for $\tan \left(\theta\left(d_{i}, J\right) / 2\right)$, yielding the following formula for $\partial \ell^{n} / \partial J$ :

$$
\frac{2 \operatorname{coth} J}{\cosh \left(d_{0} / 2\right)}\left[\sinh \left(d_{0} / 2\right) \pm \sum_{i=1}^{n-1} \sinh \left(d_{i} / 2\right) \sqrt{\frac{\sinh ^{2} J-\sinh ^{2}\left(d_{0} / 2\right)}{\sinh ^{2} J-\sinh ^{2}\left(d_{i} / 2\right)}}\right] .
$$

Here the \pm is a + if $\mathbf{d} \in \mathcal{C}_{n}$ and - otherwise. With the same dichotomy below, a similar calculation gives:

$$
-\frac{\partial \ell^{n}}{\partial d_{i}}= \pm \frac{\cosh \left(d_{i} / 2\right)}{\cosh \left(d_{0} / 2\right)} \sqrt{\frac{\sinh ^{2} J-\sinh ^{2}\left(d_{0} / 2\right)}{\sinh ^{2} J-\sinh ^{2}\left(d_{i} / 2\right)}}
$$

The formula from the statement thus follows from equations (1.3) and (1.4).

The bounds below distill a useful consequence of the formulas of Lemma 1.19 .

Proposition 1.20. For $n \geq 3$, the function $J: \mathcal{A C}_{n} \rightarrow \mathbb{R}^{+}$that records the circumcircle radius is smooth and symmetric. For $\mathbf{d} \in \mathcal{A C}_{n}$,

$$
\begin{cases}0<\left(\partial J / \partial d_{i}\right)(\mathbf{d})<1 / 2 & \text { if } \mathbf{d} \in \mathcal{C}_{n}, \text { for any } i, \\ \left(\partial J / \partial d_{i_{0}}\right)(\mathbf{d})>1 / 2 & \text { if } \mathbf{d} \in \mathcal{A C}_{n}-\left(\mathcal{C}_{n} \cup \mathcal{B C}_{n}\right) \\ & \text { and } d_{i_{0}}=\max \left\{d_{i}\right\}_{i=0}^{n-1}, \\ \left(\partial J / \partial d_{j}\right)(\mathbf{d})<0 & \text { if } \mathbf{d} \in \mathcal{A C}_{n}-\left(\mathcal{C}_{n} \cup \mathcal{B C}_{n}\right) \\ & \text { and } d_{j} \neq \max \left\{d_{i}\right\}_{i=0}^{n-1} .\end{cases}
$$

If $\mathbf{d}=\left(d_{0}, \ldots, d_{n-1}\right)$ and $d_{i}>d_{j}$, then

$$
\left|\frac{\partial J}{\partial d_{i}}(\mathbf{d})\right|>\left|\frac{\partial J}{\partial d_{j}}(\mathbf{d})\right|
$$

Proof. Circumcircle radius $J$ is symmetric by Proposition 1.14, so Lemma 1.19 actually implies that it is smooth on all of $\mathcal{A C}_{n}$. To obtain the bounds above we will fix $\mathbf{d}=\left(d_{0}, \ldots, d_{n-1}\right) \in \mathcal{A C}_{n}$ and assume that $d_{0}$ is maximal among the $d_{i}$ by rearranging if necessary. Then we will 
use equations (1.3) and (1.4) from the proof of Lemma 1.19, which hold with this hypothesis.

Applying equation (1.2) from the proof of Proposition 1.6 to equation (1.3), with $\theta_{0}(J)=\sum_{i=1}^{n-1} \theta\left(d_{i}, J\right)$, yields:

$$
\begin{aligned}
\frac{\partial J}{\partial d_{0}} & =\left[\frac{\partial \ell^{n}}{\partial J}(J)\right]^{-1} \\
& =\frac{\cosh \left(d_{0} / 2\right)}{2 \cosh J} \frac{1}{\sin \left(\theta_{0}(J) / 2\right)-\cos \left(\theta_{0}(J) / 2\right) \sum_{i=1}^{n-1} \tan \left(\theta\left(d_{i}, J\right) / 2\right)}
\end{aligned}
$$

Note that, if $\theta_{0}(J)=\pi$, then $d_{0}=2 J$ and $\partial J / \partial d_{0}=1 / 2$. We accordingly divide the remaining cases into two subcases: $\theta_{0}(J)<\pi$ and $\theta_{0}(J)>\pi$.

If $\theta_{0}(J)<\pi$, then, since $\cos \left(\theta_{0}(J) / 2\right)>0$, the denominator of the right-hand quantity above is less than $\sin \left(\theta_{0}(J) / 2\right)$. By the definition of $\ell^{n}, \sin \left(\theta_{0}(J) / 2\right)=\sinh \left(\ell^{n}(J) / 2\right) / \sinh (J)$, so since $\ell^{n}(J)=d_{0}$, we have the following inequality:

$$
\frac{\partial J}{\partial d_{0}}>\frac{\cosh \left(d_{0} / 2\right)}{2 \cosh J} \frac{\sinh J}{\sinh \left(d_{0} / 2\right)}=\frac{\tanh (J)}{2 \tanh \left(d_{0} / 2\right)}
$$

The hyperbolic tangent is increasing and $J>d_{0} / 2$, so $\partial J / \partial d_{0}>1 / 2$. This is case $\mathbf{d} \in \mathcal{A C}_{n}-\left(\mathcal{C}_{n} \cup \mathcal{B C}_{n}\right)$ by Proposition 1.17.

If $\theta_{0}(J)>\pi$, then, since $\cos \left(\theta_{0}(J) / 2\right)<0$ and $\tan x>x$ on $(0, \pi / 2)$,

$$
\begin{aligned}
\sin \left(\theta_{0}(J) / 2\right)-\cos \left(\theta_{0}(J) / 2\right) & \sum_{i=1}^{n-1} \tan \left(\theta\left(d_{i}, J\right) / 2\right) \\
& >\sin \left(\theta_{0}(J) / 2\right)-\cos \left(\theta_{0}(J) / 2\right) \cdot \pi / 2 .
\end{aligned}
$$

Since $\sin x-\cos x>1$ on $(\pi / 2, \pi)$, in this case (which, by Proposition 1.17 , is $\left.\mathbf{d} \in \mathcal{C}_{n}\right)$ we have $\partial J / \partial d_{0}<\cosh \left(d_{0} / 2\right) /(2 \cosh J)<1 / 2$.

For $i>1$, solving equation (1.4) for $\partial J / \partial d_{i}$, gives $\partial J / \partial d_{i}=$ $-\left(\partial / \partial d_{i}\right) \ell^{n}(J) /(\partial / \partial J) \ell^{n}(J)$. Let us record the numerator of this 
quantity:

$$
\begin{aligned}
\frac{\partial \ell^{n}}{\partial d_{i}}(J) & =\frac{\sinh J \cos \left(\theta_{0}(J) / 2\right)}{\cosh \left(\ell^{n}(J) / 2\right)} \\
\frac{\partial \theta}{\partial d}\left(d_{i}, J\right) & =\frac{\cosh \left(d_{i} / 2\right)}{\cosh \left(d_{0} / 2\right)} \frac{\cos \left(\theta_{0}(J) / 2\right)}{\cos \left(\theta\left(d_{i}, J\right) / 2\right)} .
\end{aligned}
$$

The second equation above follows from the definition in Lemma 1.4 after taking a partial derivative with respect to $d$. All terms here are positive, except possibly $\cos \left(\theta_{0}(J) / 2\right)$, so $\partial J / \partial d_{i}$ is negative if $\theta_{0}(J)<\pi$ (i.e., $\left.\mathbf{d} \in \mathcal{A C}_{n}-\left(\mathcal{C}_{n} \cup \mathcal{B C}_{n}\right)\right)$ and positive if $\theta_{0}(J)>\pi$, $\mathbf{d} \in \mathcal{C}_{n}$.

For fixed $J, f(d)=\cosh (d / 2) / \cos (\theta(d, J) / 2)$ is increasing since $\theta(d, J)$ increases in $d$ on $(0,2 J)$. Thus, for $i, j \geq 1$, we have $\left|\partial J / \partial d_{i}\right|>$ $\left|\partial J / \partial d_{j}\right|$ if $d_{i}>d_{j}$. We also claim that $\left|\cosh \left(d_{0} / 2\right) / \cos \left(\theta_{0}(J) / 2\right)\right| \geq$ $f\left(d_{i}\right)$. Manipulating the definition of $\ell^{n}(J)$ gives:

$$
\cos \left(\theta_{0}(J) / 2\right)= \pm \frac{\sqrt{\sinh ^{2} J-\sinh ^{2}\left(d_{0} / 2\right)}}{\sinh (J)} .
$$

By Lemma 1.4, $\cos \left(\theta\left(d_{i}, J\right) / 2\right)$ satisfies the same equation upon taking an absolute value and substituting $d_{i}$ for $d_{0}$ on the right-hand side. Since $d_{0} \geq d_{i}$, we have $\left|\cos \left(\theta_{0}(J) / 2\right)\right|<\cos \left(\theta\left(d_{i}, J\right) / 2\right)$, and the claim follows. This implies the result.

Corollary 1.21. For any $n \geq 4$ and $i, j \in\{0, \ldots, n-1\}$ such that $|i-j| \geq 2(\bmod 4)$, defining $\ell_{i, j}\left(d_{0}, \ldots, d_{n-1}\right)=\operatorname{dist}\left(x_{i}, x_{j}\right)$, where $\left\{x_{0}, \ldots, x_{n-1}\right\}$ is a cyclic $n$-gon with side length collection $\left(d_{0}, \ldots, d_{n-1}\right)$, yields a smooth function $\mathcal{A C}_{n} \rightarrow \mathbb{R}^{+}$.

Proof. Let us suppose, without loss of generality, that $i<j$, fix $\left(d_{0}, \ldots, d_{n-1}\right) \in \mathcal{A C}_{n}$, and let $\left\{x_{0}, \ldots, x_{n-1}\right\}$ be a cyclic $n$-gon with side length collection $\left(d_{0}, \ldots, d_{n-1}\right)$ and circumcircle radius $J=$ $J\left(d_{0}, \ldots, d_{n-1}\right)$. For $i_{0}$ such that $d_{i_{0}}$ is maximal, let us also assume for now that $i_{0} \notin\{i+1, \ldots, j\}$. Then, by Proposition 1.5, the angle from $x_{k-1}$ to $x_{k}$ is $\theta\left(d_{k}, J\right)<\pi$ for each $k \in\{i+1, \ldots, j\}$.

Applying Proposition 1.5, mutatis mutandis, to the cyclically or- 
dered collection $\left\{x_{i}, \ldots, x_{j}\right\}$ now gives:

$$
\ell_{i, j}\left(d_{0}, \ldots, d_{n-1}\right)=2 \sinh ^{-1}\left[\sinh J \sin \left(\frac{1}{2} \sum_{k=i+1}^{j} \theta\left(d_{k}, J\right)\right)\right] .
$$

We claim that the same formula holds near $\left(d_{0}, \ldots, d_{n-1}\right)$, whence smoothness of $\ell_{i, j}$ follows from smoothness of $J$ as well as the functions above. If it did not hold, then, for some $i_{1} \in\{i+1, \ldots, j\}$, there would be points $\left(d_{0}^{\prime}, \ldots, d_{n-1}^{\prime}\right)$ arbitrarily close to $\left(d_{0}, \ldots, d_{n-1}\right)$ such that, for a cyclic $n$-gon $\left\{x_{0}^{\prime}, \ldots, x_{n-1}^{\prime}\right\}$ on a circle of radius $J^{\prime}$ with side length collection $\left(d_{0}^{\prime}, \ldots, d_{n-1}^{\prime}\right)$, the angle from $x_{i_{1}-1}$ to $x_{i}$ is $2 \pi-\theta\left(d_{i_{1}}^{\prime}, J^{\prime}\right)$.

If this were the case, then we would have $\theta\left(d_{i_{1}}^{\prime}, J^{\prime}\right)=\sum_{i \neq i_{1}} \theta\left(d_{i}^{\prime}, J^{\prime}\right)$, for any such $\left(d_{0}^{\prime}, \ldots, d_{n-1}^{\prime}\right)$ which, upon taking a limit, would also hold for $\left(d_{0}, \ldots, d_{n-1}\right)$. But this would contradict the fact that $d_{i_{0}}$ is maximal among the $d_{i}$. This proves the claim and hence establishes the result in the case $i_{0} \notin\{i+1, \ldots, j\}$.

The case $i_{0} \in\{i+1, \ldots, j\}$ is analogous to the other, but in the formula for $\ell_{i, j}$ above, we replace the sum over $\{i+1, \ldots, j\}$ with one over $\{j+1, \ldots, n-1,0, \ldots, i\}$.

Lemma 1.22. Let $U$ be the set of points in $\left(\mathbb{R}^{+}\right)^{3}$ such that each coordinate is less than the sum of the others. For $(a, b, c) \in U$, define:

$$
\alpha(a, b, c)=\cos ^{-1}\left(\frac{\cosh b \cosh c-\cosh a}{\sinh b \sinh c}\right) .
$$

The function,

$$
A(a, b, c)=\pi-\alpha(a, b, c)-\alpha(b, c, a)-\alpha(c, a, b),
$$

is smooth on $U$. It records the hyperbolic area of a triangle in $\mathbb{H}^{2}$, with sides of length $a, b$ and $c$.

Proof. Since the inverse cosine is smooth on $(-1,1)$, the only thing to note is that the quantity in parentheses above lies in this interval, i.e., that:

$$
-\sinh b \sinh c<\cosh b \cosh c-\cosh a<\sinh b \sinh c .
$$

This follows from the angle sum identity for hyperbolic cosine and the fact that $(a, b, c) \in U$. The hyperbolic law of cosines (see [10, 
Theorem 3.5.3]) implies that $\alpha(a, b, c)$ is the angle of a hyperbolic triangle with sides of length $a, b$ and $c$ at the vertex opposite the side with length $a$. That $A(a, b, c)$ measures the area of such a triangle follows from the Gauss-Bonnet formula for hyperbolic area, see [10, Theorem 3.5.5].

Corollary 1.23. For $\mathbf{d}=\left(d_{0}, d_{1}, d_{2}\right) \in \mathcal{A C}_{3}$, define $D_{0}(\mathbf{d})=$ $A\left(d_{0}, d_{1}, d_{2}\right)$, where $A$ is as defined in Lemma 1.22. For $\mathbf{d}=$ $\left(d_{0}, d_{1}, d_{2}, d_{3}\right) \in \mathcal{A C}_{4}$, define:

$$
D_{0}(\mathbf{d})=A\left(d_{0}, d_{1}, \ell_{0,2}(\mathbf{d})\right)+A\left(\ell_{0,2}(\mathbf{d}), d_{2}, d_{3}\right) .
$$

Here $\ell_{i, j}$ is as defined in Corollary 1.21. For $n \geq 5$ and $\mathbf{d}=\left(d_{0}, \ldots\right.$, $\left.d_{n-1}\right) \in \mathcal{A C}_{n}$, define:

$$
\begin{aligned}
D_{0}(\mathbf{d})= & A\left(d_{0}, d_{1}, \ell_{0,2}(\mathbf{d})\right)+\left[\sum_{i=3}^{n-2} A\left(\ell_{0, i-1}(\mathbf{d}), d_{i}, \ell_{0, i}(\mathbf{d})\right)\right] \\
& +A\left(\ell_{0, n-2}(\mathbf{d}), d_{n-2}, d_{n-1}\right) .
\end{aligned}
$$

For each $n \geq 3$, the function $D_{0}: \mathcal{A C}_{n} \rightarrow \mathbb{R}^{+}$so defined is smooth.

Proof. Since the functions $\ell_{i, j}$ are smooth by Lemma 1.21, and the function $A$ from Lemma 1.22 is smooth on the domain $U$ described there, to establish smoothness of $D_{0}$ we must only show that each of the different inputs to $A$ that occur in its definition lie in $U$. This follows from the fact that each is the side length collection of a cyclic triangle; for instance, given $\mathbf{d}=\left(d_{0}, \ldots, d_{n-1}\right) \in \mathcal{A C}_{n}, n \geq 5$, and a cyclic $n$-gon $\left\{x_{0}, \ldots, x_{n-1}\right\}$, with side length collection $\mathbf{d},\left(\ell_{0, i-1}(\mathbf{d}), d_{i}, \ell_{0, i}(\mathbf{d})\right)$ is the side length collection of the cyclic triangle $\left\{x_{0}, x_{i-1}, x_{i}\right\}$.

If $(a, b, c)$ is the side length collection of a cyclic triangle, then $\sinh (a / 2)<\sinh (b / 2)+\sinh (c / 2)$ by Proposition 1.14. Since the hyperbolic sine has positive first and second derivatives on $(0, \infty)$, it is superadditive there, so

$$
\sinh (b / 2)+\sinh (c / 2)<\sinh ((b+c) / 2),
$$

and we conclude that $a<b+c$. Reordering and repeating this argument shows $(a, b, c) \in U$. 
2. Geometry (and more calculus). As it currently stands, following Definition 1.2, a convex cyclic n-gon is simply a sequence of points on a hyperbolic circle. Here we will first show that such a collection is the vertex set of a convex polygon in the classical sense, a finite intersection of hyperbolic half-spaces, which is compact, with area measured by the function, $D_{0}$, of Corollary 1.23.

Lemma 2.1. For $n \geq 3$, a cyclic $n$-gon $\left\{x_{0}, \ldots, x_{n-1}\right\}$ in a hyperbolic circle $C$ is the vertex set of its convex hull, a compact, convex polygon $P$ contained in the disk bounded by $C$. The edges of $P$ are the geodesic arcs $\gamma_{i}$ joining $x_{i-1}$ to $x_{i}$ for each $i>0$, together with $\gamma_{0}$ joining $x_{n-1}$ to $x_{0}$. The area of $P$ is $D_{0}\left(d_{0}, \ldots, d_{n-1}\right)$, where $d_{i}$ is the length of $\gamma_{i}$ for each $i$.

Conversely, if the vertex set of a compact, convex polygon $P$ lies in a hyperbolic circle, then enumerating it $\left\{x_{0}, \ldots, x_{n-1}\right\}$ so that with the boundary orientation from $P$, an edge points from $x_{i-1}$ to $x_{i}$ for each $i>0$, and from $x_{n-1}$ to $x_{0}$, which yields a cyclic $n$-gon in the sense of Definition 1.2.

Proof. For each $i>0$, let $\mathcal{H}_{i}$ be the half-space bounded by the geodesic through $x_{i-1}$ and $x_{i}$ such that $\mathcal{H}_{i} \cap\left[x_{i-1}, x_{i}\right]=\left\{x_{i-1}, x_{i}\right\}$. Define $\mathcal{H}_{0}$ analogously so that $\mathcal{H}_{0} \cap\left[x_{n-1}, x_{0}\right]=\left\{x_{n-1}, x_{0}\right\}$, and let

$$
P=\bigcap_{i=0}^{n-1} \mathcal{H}_{i} .
$$

Since the $\mathcal{H}_{i}$ are closed and convex in $\mathbb{H}^{2}$, so is $P$.

The criterion that $\left\{x_{0}, \ldots, x_{n-1}\right\}$ be cyclically ordered ensures for each $i$ that all $x_{j}$ other than $x_{i-1}$ and $x_{i}$ are contained in the interior of $\mathcal{H}_{i}$, since $\partial \mathcal{H}_{i}$ intersects $C$ only in $\left\{x_{i-1}, x_{i}\right\}$. In particular, $\left\{x_{0}, \ldots, x_{n-1}\right\} \subset P$. The frontier $\partial P$ of $P$ in $\mathbb{H}^{2}$ is contained in

$$
\bigcup_{i=0}^{n-1} \partial \mathcal{H}_{i},
$$

and, by the above, $\gamma_{i}=\mathcal{H}_{i} \cap \partial P$ for each $i$, since $\partial \mathcal{H}_{i}$ exits $\mathcal{H}_{i+1}$ at $x_{i}$ and $\mathcal{H}_{i-1}$ at $x_{i-1}$.

We will appeal to $[\mathbf{1 0}]$ for basic results on polygons. $P$ satisfies the definition of polygon in subsection 6.3: it is closed, convex and non- 
empty, and its collection of sides (defined in subsection 6.2) is $\left\{\gamma_{i}\right\}_{i=0}^{n-1}$. The $\gamma_{i}$ are thus its edges ("1-faces" in the notation of [10, subsection $6.3]$ ), and the $x_{i}$ are its vertices, being endpoints of the $\gamma_{i}$. Moreover, $P$ satisfies the compactness criterion of [10, Theorem 6.3.7], so by [10, Theorem 6.3.17], it is the convex hull of its vertex set. Therefore, $\left\{x_{0}, \ldots, x_{n-1}\right\}$ uniquely determines $P$, and, since it is contained in the (convex) disk bounded by $C$, so is $P$.

The diagonals from $x_{0}$ to each of $x_{2}, \ldots, x_{n-2}$ divide $P$ into a nonoverlapping union of cyclic triangles, whose area sums to the area of $P$. The diagonal lengths are $\ell_{0, i}(\mathbf{d})$ for $i \in\{2, \ldots, n-2\}$, where $\mathbf{d}=\left(d_{0}, \ldots, d_{n-1}\right)$ is the side length collection of $\left\{x_{0}, \ldots, x_{n-1}\right\}$. Therefore, Lemma 1.22 implies that $D_{0}(\mathbf{d})$ is the area of $P$.

The key observation in showing the converse statement is that, given a polygon $P$ inscribed in a circle $C$, any edge $\gamma$ of $P$ bounds a bigon outside $P$ with an arc $\gamma$ of $C$, and the initial and terminal vertices of $\gamma$ in the boundary orientation from $P$ agree with those of $\gamma$ in the counterclockwise orientation on $C$.

Below we recharacterize the "centeredness" condition from Proposition 1.17 in geometric terms, and we describe an isosceles decomposition which will be useful in analyzing $D_{0}$.

Proposition 2.2. For $n \geq 3$, a cyclic $n$-gon $\left\{x_{0}, \ldots, x_{n-1}\right\}$ is centered if and only if the center, $v$, of its circumcircle is contained in the interior of its convex hull, $P$. If this is so, then $P$ decomposes as the non-overlapping union $\bigcup_{i=0}^{n-1} T_{i}$, where $T_{i}$ is the triangle with vertices $v, x_{i}$ and $x_{i-1}$ for $i>0$ and $T_{0}$ has vertices $v, x_{0}$ and $x_{n-1}$.

If $\left\{x_{0}, \ldots, x_{n-1}\right\}$ is not centered, then $P$ has a unique longest side $\gamma_{i_{0}}$, characterized by the fact that the geodesic containing $\gamma_{i_{0}}$ has $v$ and $P$ in opposite half-spaces. In this case, $P \cap T_{i_{0}}=\gamma_{i_{0}}$, and $P \cup T_{i_{0}}$ is a convex polygon that decomposes as the non-overlapping union $\bigcup_{i \neq i_{0}} T_{i}$.

If $\left\{x_{0}, \ldots, x_{n-1}\right\}$ is semicyclic, then $v$ is the midpoint of $\gamma_{i_{0}}, T_{i_{0}}=$ $\gamma_{i_{0}}$, and also

$$
P=\bigcup_{i=0}^{n-1} T_{i} .
$$


Proof. Let $\left\{x_{0}, \ldots, x_{n-1}\right\}$ have side length collection $\left(d_{0}, \ldots, d_{n-1}\right)$. Lemma 1.4 implies that the triangle $T_{i}$ defined above has interior angle $\theta\left(d_{i}, J\right) \in(0, \pi]$ at $v$, where $J$ is the radius of the circumcircle $C$. The edge of $T_{i}$ opposite $v$, which is the geodesic arc $\gamma_{i}$ joining $x_{i}$ to $x_{i-1}$, divides $C$ into two arcs: $\left[x_{i-1}, x_{i}\right]$ and $\left[x_{i}, x_{i-1}\right]$ in the notation of Definition 1.2. If $T_{i}$ is not degenerate, i.e., if $\theta\left(d_{i}, J\right) \neq \pi$, then the shorter of these arcs lies on the opposite side of the geodesic $\delta_{i}$ containing $\gamma_{i}$ from $v$.

It follows that, for each $i$ such that the angle from $x_{i-1}$ to $x_{i}$ is less than $\pi$, it is $\theta\left(d_{i}, J\right)$, and $v$ lies in the interior of the half-space $\mathcal{H}_{i}$ bounded by $\delta_{i}$ that intersects $\left[x_{i-1}, x_{i}\right]$ only in $\left\{x_{i-1}, x_{i}\right\}$. If the angle from $x_{i-1}$ to $x_{i}$ is greater than $\pi$, then it is $2 \pi-\theta\left(d_{i}, J\right)$, and $v$ lies in the interior of the half-space $\mathcal{H}_{i}^{\prime}$ bounded by $\delta_{i}$ opposite $\mathcal{H}_{i}$. The angle from $x_{i-1}$ to $x_{i}$ is $\pi$ if and only if $v$ is the midpoint of $\gamma_{i}$ (the degenerate case mentioned above), so in this case, $v$ is in $\mathcal{H}_{i} \cap \mathcal{H}_{i}^{\prime}$ but in the interior of neither.

Recall from Proposition 1.17 that $\left\{x_{0}, \ldots, x_{n-1}\right\}$ is centered if and only if the angle from $x_{i-1}$ to $x_{i}$ is less than $\pi$ for each $i$. By the paragraph above, this holds if and only if $v$ is in the interior of $P=\bigcap_{i=0}^{n-1} \mathcal{H}_{i}$ (compare the proof of Lemma 2.1). In this case, the decomposition of $P$ as a non-overlapping union of $T_{i}$ is obtained by simply coning from $v$ to $\partial P$ (which, by Lemma 2.1, is the union of the $\gamma_{i}$ ). Again, by Proposition 1.17, $\left\{x_{0}, \ldots, x_{n-1}\right\}$ is semicyclic if and only if $J=d_{i_{0}} / 2$, where $d_{i_{0}}$ is maximal among the $d_{i}$. In this case, $\gamma_{i_{0}}$ is a diameter of $C$, so $v$ is its midpoint and $T_{i_{0}}=\gamma_{i_{0}}$ as claimed. Again, coning from $v$, gives $P=\bigcup T_{i}$.

If the angle from $x_{i_{0}-1}$ to $x_{i_{0}}$ is at least $\pi$ for some $i_{0}$, this $i_{0}$ is unique since the angles from $x_{i-1}$ to $x_{i}$ sum to $2 \pi\left(\left\{x_{0}, \ldots, x_{n-1}\right\}\right.$ being cyclically ordered). Therefore, this angle is $2 \pi-\theta\left(d_{i_{0}}, J\right)$; for all other $i$, the angle from $x_{i-1}$ to $x_{i}$ is $\theta\left(d_{i}, J\right)<\pi$, and

$$
\theta\left(d_{i_{0}}, J\right)=\sum_{i \neq i_{0}} \theta\left(d_{i}, J\right)
$$

In particular, $\theta\left(d_{i_{0}}, J\right)>\theta\left(d_{i}, J\right)$, so $d_{i_{0}}>d_{i}$ for all $i \neq i_{0}$. In this case, $v$ lies in $\mathcal{H}_{i_{0}}^{\prime}$; hence, so do $T_{i_{0}}$ and $T_{i_{0}} \cap P=\gamma_{i_{0}}$. But, $v$ is in the interior of $\mathcal{H}_{i}$ for all $i \neq i_{0}$. 
Because the angle from $x_{i_{0}-1}$ to $x_{i_{0}}$ is at least $\pi$, the diameters of $C$ through $x_{i_{0}-1}$ and $x_{i_{0}}$ bound half-spaces $\mathcal{H}_{i_{0}-1}^{\prime}$ and $\mathcal{H}_{i_{0}}^{\prime}$, respectively, which contain all $x_{i}$, and hence, $P$. It follows as in the proof of Lemma 2.1 that

$$
P \cup T_{i_{0}}=\mathcal{H}_{i_{0}-1}^{\prime} \cap \mathcal{H}_{i_{0}}^{\prime} \cap \bigcap_{i \neq i_{0}} \mathcal{H}_{i}
$$

is a convex polygon, and its decomposition as $\bigcup_{i \neq i_{0}} T_{i}$ follows by coning from $v$.

Proposition 2.3. For $n \geq 3$, the function $D_{0}: \mathcal{A C}_{n} \rightarrow \mathbb{R}^{+}$that records hyperbolic area is smooth and symmetric. Its partial derivative $\partial D_{0} / \partial d_{i}$ with respect to $d_{i}$ is given at $\mathbf{d}=\left(d_{0}, \ldots, d_{n-1}\right) \in \mathcal{A C}_{n}$ by:

$$
\begin{cases}-\sqrt{\left[1 / \cosh ^{2}\left(d_{i} / 2\right)\right]-\left[1 / \cosh ^{2} J(\mathbf{d})\right]} & \text { if } \mathbf{d} \in \mathcal{A C}_{n}-\mathcal{C}_{n} \text { and } \\ & d_{i}=\max \left\{d_{j}\right\}_{j=0}^{n-1}, \\ \sqrt{\left[1 / \cosh ^{2}\left(d_{i} / 2\right)\right]-\left[1 / \cosh ^{2} J(\mathbf{d})\right]} & \text { otherwise. }\end{cases}
$$

Proof. We have already shown in Corollary 1.23 that $D_{0}$ is smooth and, in Lemma 2.1, that, for a given $\mathbf{d}=\left(d_{0}, \ldots, d_{n-1}\right) \in \mathcal{A C}_{n}, D_{0}(\mathbf{d})$ is the area of the convex hull of a cyclic $n$-gon with side length collection $\left(d_{0}, \ldots, d_{n-1}\right)$. Using the decomposition of Proposition 2.2 and taking $J=J(\mathbf{d})$ and $d_{i_{0}}=\max \left\{d_{i}\right\}$, we can rewrite its formula as follows:

$$
D_{0}(\mathbf{d})= \begin{cases}\sum_{i=0}^{n-1} A\left(J, J, d_{i}\right) & \text { if } \mathbf{d} \in \mathcal{C}_{n}, \\ \left(\sum_{i \neq i_{0}} A\left(J, J, d_{i}\right)\right)-A\left(J, J, d_{i_{0}}\right) & \text { otherwise. }\end{cases}
$$

This follows from Lemma 1.22 and the fact that each of the triangles $T_{i}$ of Proposition 2.2 is isosceles, with two sides of length $J(\mathbf{d})$ and one of length $d_{i}$. It implies that $D_{0}$ is symmetric in $\left(d_{0}, \ldots, d_{n-1}\right)$.

Comparing the function $\alpha$ of Lemma 1.22 with $\theta$ from Lemma 1.4, we note that $\alpha(d, J, J)=\theta(d, J)$ for any $d<2 J$. Moreover, a little hyperbolic trigonometry shows that

$$
\alpha(J, d, J)=\alpha(J, J, d)=\cos ^{-1}(\operatorname{coth} J \tanh (d / 2)) .
$$


These facts and some trigonometric identities can be used to show the following:

$$
\cos \left(A\left(J, J, d_{i}\right) / 2\right)=\frac{\cosh ^{2}\left(d_{i} / 2\right)+\cosh J}{\cosh \left(d_{i} / 2\right)(\cosh J+1)} .
$$

Taking derivatives and doing some more trigonometry, we obtain the following formulas for $\partial A / \partial d_{j}\left(J, J, d_{i}\right)$ :

$$
\left\{\begin{array}{c}
(2 \sinh J / \cosh J+1)\left[\sinh \left(d_{i} / 2\right)\right] /\left[\sqrt{\sinh ^{2} J-\sinh ^{2}\left(d_{i} / 2\right)}\right]\left(\partial J / \partial d_{j}\right) \\
j \neq i, \\
(2 \sinh J / \cosh J+1)\left[\sinh \left(d_{j} / 2\right)\right] /\left[\sqrt{\sinh ^{2} J-\sinh ^{2}\left(d_{j} / 2\right)}\right]\left(\partial J / \partial d_{j}\right) \\
-\left[\cosh ^{2}\left(d_{j} / 2\right)-\cosh J\right] /\left[\cosh \left(d_{j} / 2\right) \sqrt{\sinh ^{2} J-\sinh ^{2}\left(d_{j} / 2\right)}\right] \\
j=i .
\end{array}\right.
$$

Note that this formula is only defined for $d_{i}<2 J$. This is because the inverse cosine is smooth only on $(-1,1)$. We will use it below to compute partial derivatives of $D_{0}$ at $\mathbf{d}=\left(d_{0}, \ldots, d_{n-1}\right) \in \mathcal{A C}_{n}$, but, due to this issue, we will assume that $J(\mathbf{d})<\max \left\{d_{i}\right\}$, i.e., that $\mathbf{d} \notin \mathcal{B C}_{n}$ (recall Proposition 1.17). Since $\mathcal{B C}_{n}$ is a codimensionone submanifold (Proposition 1.18), values there are determined by continuity.

Using symmetricity of $D_{0}$, we will assume below that $d_{0}$ is maximal among the $d_{i}$. Applying the formula of Lemma 1.19, we divide out a factor of $\sqrt{\sinh ^{2} J-\sinh ^{2}\left(d_{0} / 2\right)}$. This gives the formula for $\partial J / \partial d_{j}(\mathbf{d})$ below:

$$
\begin{aligned}
& \frac{ \pm(1 / 2) \cosh \left(d_{j} / 2\right) \tanh J}{\sqrt{\sinh ^{2} J-\sinh ^{2}\left(d_{j} / 2\right)\left[\sinh \left(d_{0} / 2\right)\right] /\left[\sqrt{\sinh ^{2} J-\sinh ^{2}\left(d_{0} / 2\right)}\right]}} \\
& \cdot \frac{ \pm(1 / 2) \cosh \left(d_{j} / 2\right) \tanh J}{ \pm \sum_{i=1}^{n-1}\left[\sinh \left(d_{i} / 2\right)\right] /\left[\sqrt{\sinh ^{2} J-\sinh ^{2}\left(d_{i} / 2\right)}\right]} .
\end{aligned}
$$

We recall from Lemma 1.19 that each \pm above should be read as + if $\mathbf{d} \in \mathcal{C}_{n}$ and - if $\mathbf{d} \in \mathcal{A} \mathcal{C}_{n}-\mathcal{C}_{n}$, except that the numerator is positive in the latter case for $j=0$. 
We finally come to the derivative computation. We use the formula above for $D_{0}$ and consider three cases. For the first, $\mathbf{d} \in \mathcal{C}_{n}$, and we have:

$$
\begin{aligned}
\frac{\partial}{\partial d_{j}} D_{0}(\mathbf{d})= & \frac{2 \sinh J}{\cosh J+1} \frac{\partial J}{\partial d_{j}}\left(\sum_{i=0}^{n-1} \frac{\sinh \left(d_{i} / 2\right)}{\sqrt{\sinh ^{2} J-\sinh ^{2}\left(d_{i} / 2\right)}}\right) \\
& -\frac{\cosh ^{2}\left(d_{j} / 2\right)-\cosh J}{\cosh \left(d_{j} / 2\right) \sqrt{\sinh ^{2} J-\sinh ^{2}\left(d_{j} / 2\right)}} \\
= & \frac{1}{\sqrt{\sinh ^{2} J-\sinh ^{2}\left(d_{j} / 2\right)}}\left[\frac{\sinh ^{2} J \cosh \left(d_{j} / 2\right)}{\cosh J(\cosh J+1)}-\frac{\cosh ^{2}\left(d_{j} / 2\right)-\cosh J}{\cosh \left(d_{j} / 2\right)}\right] \\
= & \frac{1}{\sqrt{\sinh ^{2} J-\sinh ^{2}\left(d_{j} / 2\right)}}\left[\frac{\cosh ^{2} J-\cosh ^{2}\left(d_{j} / 2\right)}{\cosh J \cosh \left(d_{j} / 2\right)}\right] \\
= & \frac{\sqrt{\cosh ^{2} J-\cosh \left(d_{j} / 2\right)}}{\cosh J \cosh \left(d_{j} / 2\right)}
\end{aligned}
$$

For $\mathbf{d} \in \mathcal{A C}_{n}-\mathcal{C}_{n}$, we first treat the case $\partial D_{0} / \partial d_{j}$ for $j>0$, where:

$$
\begin{array}{r}
\frac{\partial}{\partial d_{j}} D_{0}(\mathbf{d})=\frac{2 \sinh J}{\cosh J+1} \frac{\partial J}{\partial d_{j}}\left[\left(\sum_{i=1}^{n-1} \frac{\sinh \left(d_{i} / 2\right)}{\sqrt{\sinh ^{2} J-\sinh ^{2}\left(d_{i} / 2\right)}}\right)\right. \\
\left.-\frac{\sinh \left(d_{0} / 2\right)}{\sqrt{\sinh ^{2} J-\sinh ^{2}\left(d_{0} / 2\right)}}\right]-\frac{\cosh ^{2}\left(d_{j} / 2\right)-\cosh J}{\cosh \left(d_{j} / 2\right) \sqrt{\sinh ^{2} J-\sinh ^{2}\left(d_{j} / 2\right)}} \\
=\frac{\sqrt{\cosh ^{2} J-\cosh \left(d_{j} / 2\right)}}{\cosh J \cosh \left(d_{j} / 2\right)} .
\end{array}
$$

Intermediate steps parallel the previous computation. Finally, again for $\mathbf{d} \in \mathcal{A C}_{n}-\mathcal{C}_{n}$,

$$
\begin{aligned}
\frac{\partial}{\partial d_{0}} D_{0}(\mathbf{d})=\frac{2 \sinh J}{\cosh J+1} \frac{\partial J}{\partial d_{0}}[ & \left(\sum_{i=1}^{n-1} \frac{\sinh \left(d_{i} / 2\right)}{\sqrt{\sinh ^{2} J-\sinh ^{2}\left(d_{i} / 2\right)}}\right) \\
& \left.-\frac{\sinh \left(d_{0} / 2\right)}{\sqrt{\sinh ^{2} J-\sinh ^{2}\left(d_{0} / 2\right)}}\right]
\end{aligned}
$$




$$
\begin{aligned}
& +\frac{\cosh ^{2}\left(d_{0} / 2\right)-\cosh J}{\cosh \left(d_{0} / 2\right) \sqrt{\sinh ^{2} J-\sinh ^{2}\left(d_{0} / 2\right)}} \\
& =-\frac{\sqrt{\cosh ^{2} J-\cosh \left(d_{j} / 2\right)}}{\cosh J \cosh \left(d_{0} / 2\right)} .
\end{aligned}
$$

This proves the result.

Corollary 2.4. For $n \geq 3, \mathbf{d}=\left(d_{0}, \ldots, d_{n-1}\right)$ and $\mathbf{d}^{\prime}=\left(d_{0}^{\prime}, \ldots, d_{n-1}^{\prime}\right)$ in $\mathcal{C}_{n} \cup \mathcal{B C}_{n}$ if, after a permutation $d_{i} \leq d_{i}^{\prime}$ for all $i$, and $d_{i}<d_{i}^{\prime}$ for some $i$, then $D_{0}(\mathbf{d})<D_{0}\left(\mathbf{d}^{\prime}\right)$.

Proof. Given such $\left(d_{0}, \ldots, d_{n-1}\right)$ and $\left(d_{0}^{\prime}, \ldots, d_{n-1}^{\prime}\right)$ in $\mathcal{C}_{n} \cup \mathcal{B C}_{n}$, since $D_{0}$ is symmetric, we may assume $d_{i} \leq d_{i}^{\prime}$ for each $i$. Furthermore, we will take $d_{0}$ maximal among the $d_{i}$. We will produce a path $\left(d_{0}(t), \ldots, d_{n-1}(t)\right)$ from $\left(d_{0}, \ldots, d_{n-1}\right)$ to $\left(d_{0}^{\prime}, \ldots, d_{n-1}^{\prime}\right)$, with its interior in $\mathcal{C}_{n}$, that is piecewise-smooth and has each $d_{i}(t)$ non-decreasing. The result will thus follow directly from the chain rule and Proposition 2.3. Then,

In defining the path, we will take $d=\min _{i}\left\{d_{i}\right\}$ and $D=\max _{i}\left\{d_{i}^{\prime}\right\}$.

$$
d_{i}(t)= \begin{cases}d_{i} & d+t \leq d_{i} \\ d+t & d_{i} \leq d+t \leq d_{i}^{\prime} \\ d_{i}^{\prime} & \text { otherwise }\end{cases}
$$

for $0 \leq t \leq D-d$. It is clear by inspection that $d_{i}(t)$ is nondecreasing and piecewise-smooth. Thus it remains only to check that $\left(d_{0}(t), \ldots, d_{n-1}(t)\right) \in \mathcal{C}_{n}$. We break this up into cases.

For $0 \leq t \leq d_{0}-d, d_{0}=d_{0}(t)$ is maximal among the $d_{i}(t)$ by construction, since $d_{0}$ is maximal among the $d_{i}$. Here, we have:

$$
\sum_{i=0}^{n-1} \theta\left(d_{i}(t), d_{0}(t) / 2\right)=\sum_{i=0}^{n-1} \theta\left(d_{i}(t), d_{0} / 2\right)>\sum_{i=0}^{n-1} \theta\left(d_{i}, d_{0} / 2\right) \geq 2 \pi .
$$

The first inequality above follows from the fact that $\theta(d, J)$ (introduced in Lemma 1.4) increases in $d$. The second follows from Proposition 1.17 and the hypothesis that $\left(d_{0}, \ldots, d_{n-1}\right) \in \mathcal{C}_{n} \cup \mathcal{B C}_{n}$, which thus also implies that $\left(d_{0}(t), \ldots, d_{n-1}(t)\right) \in \mathcal{C}_{n}$. 
If $d_{0}=D$, then the above case completes the proof. So assume $D>d_{0}$. Let $i_{0} \in\{0, \ldots, n-1\}$ be such that $D=d_{i_{0}}^{\prime}$, and let $d_{i_{1}}^{\prime}$ be maximal among the $d_{i}^{\prime}$ with $i \neq i_{0}$.

First suppose that $d_{0}<d_{i_{1}}^{\prime}$. Then, for $d_{0}-d \leq t \leq d_{i_{1}}^{\prime}-d$, at least $d_{i_{0}}(t)$ and $d_{i_{1}}(t)$, take the maximum value $d+t$ among the entries $d_{i}(t)$, so:

$$
\sum_{i=0}^{n-1} \theta\left(d_{i}(t),(d+t) / 2\right)=2 \pi+\sum_{i \neq i_{0}, i_{1}} \theta\left(d_{i}(t),(d+t) / 2\right)>2 \pi .
$$

Thus, $\left(d_{0}(t), \ldots, d_{n-1}(t)\right) \in \mathcal{C}_{n}$ for $d_{0}-d \leq t \leq d_{i_{1}}^{\prime}-d$.

We finally consider the interval $d_{i_{1}}^{\prime}-d \leq t<D-d$. Note that, if $d_{0} \geq d_{i_{1}}^{\prime}$, then this and the interval $0 \leq t \leq d_{0}-d$ cover the domain of the $d_{i}(t)$, and we can skip the case above. On the other hand, if $d_{i_{1}}^{\prime}=d_{i_{0}}^{\prime}$, then the previous two cases cover the entire domain, and we are done.

Let us therefore assume that $d_{i_{1}}^{\prime}<d_{i_{0}}^{\prime}=D$. Then, on the interval in question, $d_{i}(t)=d_{i}^{\prime}$ for each $i \neq i_{0}$, and $d_{i_{0}}(t)=d+t$ is the unique maximal $d_{i}(t)$. For $t<D-d$, we have:

$$
\sum_{i=0}^{n-1} \theta\left(d_{i}(t), d_{i_{0}}(t) / 2\right)=\pi+\sum_{i \neq i_{0}} \theta\left(d_{i}^{\prime}, d_{i_{0}}(t) / 2\right)>\pi+\sum_{i \neq i_{0}} \theta\left(d_{i}^{\prime}, d_{i_{0}}^{\prime} / 2\right) .
$$

The final quantity is at least $2 \pi$, so $\left(d_{0}(t), \ldots, d_{n-1}(t)\right) \in \mathcal{C}_{n}$ for $d_{i_{1}}^{\prime}-d \leq t<D-d$, and the result is proved.

\section{To infinity...}

Definition 3.1. Let $\overline{\mathbb{H}}^{2}$ be the closure of the upper half-plane $\mathbb{H}^{2}$ in the one-point compactification $\mathbb{C} \cup\{\infty\}$ of $\mathbb{C}$. The ideal boundary of $\mathbb{H}^{2}$ is $\overline{\mathbb{H}}^{2}-\mathbb{H}^{2}=\mathbb{R} \cup\{\infty\}$.

In this section, we will show that $\mathcal{A C}_{n}$, which parametrizes cyclic hyperbolic $n$-gons, has as its frontier in $(0, \infty)^{n}$ a space $\mathcal{H C}_{n}$ that parametrizes horocyclic $n$-gons, those with vertices on a "horocycle" (defined below). We will also describe horocyclic ideal $n$-gons, which are natural limits for certain families of cyclic $n$-gons with edge lengths approaching infinity. 
Definition 3.2. Let $C_{\infty}=\mathbb{R}+i$ and $B_{\infty}=\{z \in \mathbb{C} \mid \Im z \geq 1\}$, and note that, in $\mathbb{C} \cup\{\infty\},\{\infty\}=\overline{C_{\infty}}-C_{\infty}$. A horocycle of $\mathbb{H}^{2}$ is a $\operatorname{PSL}(2, \mathbb{R})$-translate of $C_{\infty}$, and its ideal point and the horoball that it bounds are the corresponding translates of $\infty$ and $B_{\infty}$, respectively.

The action of $\mathrm{PSL}_{2}(\mathbb{R})$ is transitive on $\mathbb{R} \cup\{\infty\}$, the stabilizer of $\infty$ acts transitively on horizontal lines in $\mathbb{H}^{2}$ via $\left\{\left(\begin{array}{cc}r & 0 \\ 0 & 1\end{array}\right)\right\}$, and the stabilizer $\left\{\left(\begin{array}{ll}1 & r \\ 0 & 1\end{array}\right)\right\}$ of $C_{\infty}$ acts transitively on it.

The horocycles are thus the horizontal straight lines and the nonempty intersections with $\mathbb{H}^{2}$ of circles tangent to $\mathbb{R}$. The horoball that one bounds is the region above the line in the former case and inside the circle in the latter. So it is natural to orient horocycles counterclockwise by giving them the boundary orientation from their horoballs.

Definition 3.3. If $C$ is a horocycle with ideal point $v$, then $C \cup\{v\}$ is a circle. We may thus define the notions of counterclockwise and cyclic order by analogy with Definition 1.2.

A horocyclic $n$-gon is a collection $\left\{x_{0}, \ldots, x_{n-1}\right\}$ of distinct points on a horocycle $C$ with ideal point $v$ that is cyclically ordered on $C \cup\{v\}$. A horocyclic ideal $n$-gon is a cyclically ordered collection $\left\{x_{0}, \ldots, x_{n-1}\right\}$ on some $C \cup\{v\}$ with an ideal vertex $x_{i}=v$.

The side length collection of a horocyclic $n$-gon $\left\{x_{0}, \ldots, x_{n-1}\right\}$ is $\left(d_{0}, \ldots, d_{n-1}\right)$, where $d_{i}=\operatorname{dist}\left(x_{i-1}, x_{i}\right)$ for each $i>0$ and $d_{0}=$ $\operatorname{dist}\left(x_{0}, x_{n-1}\right)$. It is defined analogously for a horocyclic ideal $n$-gon $\left\{x_{0}, \ldots, x_{n-1}\right\}$, except that $d_{i}=\infty$ if $x_{i}$ or $x_{i-1}$ is the ideal vertex.

Proposition 3.4. For $n \geq 3,\left(d_{0}, \ldots, d_{n-1}\right) \in(0, \infty)^{n}$ is the side length collection of a horocyclic $n$-gon if and only if $\sinh \left(d_{i} / 2\right)=$ $\sum_{j \neq i} \sinh \left(d_{j} / 2\right)$ for some $i$, and $\left(d_{0}, \ldots, d_{n-1}\right) \in(0, \infty]^{n}$ is the side length collection of a horocyclic ideal n-gon if and only if $d_{i_{0}}=d_{i_{0}+1}=$ $\infty$ for a unique $i_{0}$ (taking $i_{0}+1=0$ if $i_{0}=n-1$ ). Two horocyclic or horocyclic ideal n-gons are isometric if and only if their side length collections differ by a cyclic permutation.

Proof. Since the isometry group of $\mathbb{H}^{2}$ acts transitively on horocycles, given a horocyclic $n$-gon, $\left\{x_{0}, \ldots, x_{n-1}\right\}$, we may assume it lies on $C_{\infty}$. The key fact here follows from a short explicit calcula- 
tion (or an appeal to, e.g., [5, Theorem 1.2.6 (iii)]): for $x, y \in C_{\infty}$, $\sinh (\operatorname{dist}(x, y) / 2)=|x-y| / 2$.

For the unique $i$ such that the counterclockwise arc from $x_{i-1}$ to $x_{i}$ contains $\{\infty\}$, the real coordinate of $x_{i}$ is minimal among the $x_{j}$, and real coordinates increase along the sequence $x_{i}, \ldots, x_{n-1}, x_{0}, \ldots, x_{i-1}$. The key fact thus implies that

$$
\sinh \left(d_{i} / 2\right)=\sum_{j \neq i} \sinh \left(d_{j} / 2\right)
$$

On the other hand, given $\left(d_{0}, \ldots, d_{n-1}\right)$ such that $\sinh \left(d_{i} / 2\right)=$ $\sum_{j \neq i} \sinh \left(d_{j} / 2\right)$, a collection $\left\{x_{0}, \ldots, x_{n-1}\right\}$ on $C_{\infty}$, laid out in the order above, has side length collection $\left(d_{0}, \ldots, d_{n-1}\right)$, if the real coordinates of successive points $x_{i-1}$ and $x_{i}$ differ by $\ell_{i}=2 \sinh \left(d_{i} / 2\right)$.

It is clear that the condition for $\left(d_{0}, \ldots, d_{n-1}\right) \in(0, \infty]^{n}$ to be the side length collection of a horocyclic ideal $n$-gon is necessary. Existence follows the strategy above: we put $x_{i_{0}}$ at $\infty$ for the unique $i_{0}$ such that $d_{i_{0}}=d_{i_{0}+1}=\infty$ and arrange $x_{i_{0}+1}, \ldots, x_{n-1}, x_{0}, \ldots, x_{i_{0}-1}$ on $C_{\infty}$ with real coordinates in increasing order.

Corollary 3.5. For each $n \geq 3$, the set of marked, horocyclic $n$-gons in $\mathbb{H}^{2}$ is parametrized by the frontier of $\mathcal{A C}_{n}$ (defined in Corollary 1.16) in $(0, \infty)^{n}$. Taking $\mathbf{d}=\left(d_{0}, \ldots, d_{n-1}\right)$ below, this is:

$\mathcal{H C}_{n}=\left\{\mathbf{d} \mid \sinh \left(d_{i_{0}} / 2\right)=\sum_{i \neq i_{0}} \sinh \left(d_{i_{0}} / 2\right)\right.$, where $\left.d_{i_{0}}=\max \left\{d_{i}\right\}_{i=0}^{n-1}\right\}$

It is the orbit of graph $\left(h_{0}\right) \doteq\left\{\left(h_{0}(\mathbf{d}), \mathbf{d}\right) \mid \mathbf{d} \in\left(\mathbb{R}^{+}\right)^{n-1}\right\}$ under cyclic permutation of entries, where

$$
h_{0}(\mathbf{d})=2 \sinh ^{-1}\left(\sum_{i=1}^{n-1} \sinh \left(d_{i} / 2\right)\right)
$$

is symmetric, smooth and strictly increasing in each variable. Moreover, $h_{0}(\mathbf{d})>b_{0}(\mathbf{d})$ for each $\mathbf{d} \in\left(\mathbb{R}^{+}\right)^{n-1}$, where $b_{0}$ is as in Proposition 1.18 , so $\mathcal{H C}_{n}$ has a neighborhood disjoint from $\mathcal{C}_{n} \cup \mathcal{B C}_{n}$ in $(0, \infty)^{n}$. 
The set of marked, horocyclic ideal n-gons is parametrized by:

$$
\mathcal{H} \mathcal{I}_{n}=\left\{\mathbf{d} \mid d_{i_{0}}=d_{i_{0}+1}=\infty \text { for a unique } i_{0}, 0 \leq i_{0}<n\right\} .
$$

It is the orbit of $\{(\infty, \infty)\} \times \mathbb{R}^{n-2}$ under cyclic permutation of entries.

Proof. Most of the above is obvious and/or a consequence of Proposition 3.4, but we will prove that $h_{0}(\mathbf{d})>b_{0}(\mathbf{d})$. Recall from Proposition 1.18 that $b_{0}(\mathbf{d})$ is defined by the equation $\sum_{i=1}^{n-1} \theta\left(d_{i}, b_{0}(\mathbf{d}) / 2\right)=\pi$, for $\theta$ as in Lemma 1.4. Plugging into $\ell^{n}$ from Proposition 1.5 gives $\ell^{n}\left(b_{0}(\mathbf{d}) / 2, \mathbf{d}\right)=b_{0}(\mathbf{d})$. The inequality thus follows from the fact that fixing $\mathbf{d}, \ell^{n}$ increases in $J$ to a limit of $h_{0}(\mathbf{d})$, by Proposition 1.6.

Proposition 3.6. For $n \geq 3$, values of the circumcircle radius function $J$ approach $\infty$ on a sequence in $\mathcal{A C}_{n}$ approaching $\mathcal{H C}_{n}$ or $\mathcal{H I}_{n}$.

Proof. Suppose $\mathbf{d}=\left(d_{0}, \ldots, d_{n-1}\right) \in \mathcal{H C}_{n}$ is approached by a sequence in $\mathcal{A C}_{n}$. The unique maximal entry of $\mathbf{d}$ is $d_{i_{0}}$ such that $\sinh \left(d_{i_{0}} / 2\right)=\sum_{i \neq i_{0}} \sinh \left(d_{i} / 2\right)$, so the $i_{0}$ entry is also maximal for all but finitely many points in the sequence. Using symmetricity of $J$, we will assume that $i_{0}=0$, so Lemma 1.15 implies that $d_{0}=\ell^{n}(J)$ for each point in the sequence.

If it is not true that $J(\mathbf{d}) \rightarrow \infty$, then upon passing to a subsequence we could ensure that $J(\mathbf{d}) \rightarrow J_{0}$ for some real $J_{0}$. But $\ell^{n}$ is continuous as a function of $\left(d_{1}, \ldots, d_{n-1}, J\right)$ such that $J \geq 2 \max \left\{d_{i}\right\}$ (cf., Proposition 1.6), so since the initial coordinate also converges this would imply that $d_{0}=\ell^{n}\left(J_{0}, d_{1}, \ldots, d_{n-1}\right)$ at $\mathbf{d} \in \mathcal{H C}_{n}$. But this is not possible since $\ell^{n}$ increases toward its asymptote, which is $d_{0}=h_{0}\left(d_{1}, \ldots, d_{n-1}\right)$ (recall Proposition 1.6).

That values of $J$ approach infinity on a sequence approaching $\mathcal{H} \mathcal{I}_{n}$ is an immediate consequence of the fact that $J\left(d_{0}, \ldots, d_{n-1}\right) \geq$ $\max \left\{d_{i}\right\} / 2$.

Proposition 3.7. For $n \geq 3$, the formulas below define a symmetric, continuous extension of $D_{0}$ to $\mathcal{A C}_{n} \cup \mathcal{H C}_{n} \cup \mathcal{H} \mathcal{I}_{n}$. For $\mathbf{d}=$ 
$\left(d_{0}, \ldots, d_{n-1}\right) \in \mathcal{H C}_{n}$ with maximal entry $d_{i_{0}}$, define:

$D_{0}(\mathbf{d})=(n-2) \pi+2\left[\sin ^{-1}\left(\frac{1}{\cosh \left(d_{i_{0}} / 2\right)}\right)-\sum_{i \neq i_{0}} \sin ^{-1}\left(\frac{1}{\cosh \left(d_{i} / 2\right)}\right)\right]$.

For $\mathbf{d}=\left(d_{0}, \ldots, d_{n-1}\right) \in \mathcal{H} \mathcal{I}_{n}$ with $d_{i_{0}}=d_{i_{0}+1}=\infty$, take

$$
D_{0}(\mathbf{d})=(n-2) \pi-2 \sum_{i \neq i_{0}, i_{0}+1} \sin ^{-1}\left(\frac{1}{\cosh \left(d_{i} / 2\right)}\right) \text {. }
$$

Given $\left(d_{0}, \ldots, d_{n-1}\right)$ and $\left(d_{0}^{\prime}, \ldots, d_{n-1}^{\prime}\right)$ in $\mathcal{H C}_{n} \cup \mathcal{H I}_{n}$, if, up to a fixed permutation $d_{i} \leq d_{i}^{\prime}$ for each $i$, and $d_{i}<d_{i}^{\prime}$ for some $i$, then $D_{0}\left(d_{0}, \ldots, d_{n-1}\right)<D_{0}\left(d_{0}^{\prime}, \ldots, d_{n-1}^{\prime}\right)$.

Proof. Both cases of this result follow from:

Claim 3.8. For sequences $\left\{a_{k}\right\},\left\{b_{k}\right\}$ and $\left\{c_{k}\right\}$ of positive real numbers such that $a_{k} \rightarrow a \in(0, \infty)$ and $b_{k} \rightarrow \infty$ as $k \rightarrow \infty$, and $\mid \sinh \left(c_{k} / 2\right)-$ $\sinh \left(b_{k} / 2\right) \mid<\sinh \left(a_{k} / 2\right)$, for all $k$, the area function $A$ of Lemma 1.22 satisfies:

$$
\lim _{k \rightarrow \infty} A\left(a_{k}, b_{k}, c_{k}\right)=\pi-2 \sin ^{-1}\left(\frac{1}{\cosh (a / 2)}\right)
$$

This claim is a consequence of the definition of $A$ and the following limit computation:

$$
\begin{aligned}
\lim _{k \rightarrow \infty} \alpha\left(a_{k}, b_{k}, c_{k}\right) & =0 \\
\lim _{k \rightarrow \infty} \alpha\left(b_{k}, c_{k}, a_{k}\right) & =\lim _{k \rightarrow \infty} \alpha\left(c_{k}, a_{k}, b_{k}\right) \\
& =\cos ^{-1}\left(\frac{\cosh a-1}{\sinh a}\right)=\sin ^{-1}\left(\frac{1}{\cosh (a / 2)}\right) .
\end{aligned}
$$

This in turn is a calculus exercise using the definition of $\alpha(a, b, c)$ (again in Lemma 1.22).

In the case that a sequence approaches $\left(d_{0}, \ldots, d_{n-1}\right) \in \mathcal{H C}_{n}$, by Corollary 3.5, we may assume that all but finitely many terms lie in $\mathcal{A C}_{n}-\mathcal{C}_{n}$ with maximal $i_{0}$ entry, where $d_{i_{0}}$ is maximal among the $d_{i}$. We therefore apply the claim to formula (2.1), with $b_{k}=c_{k}=J$. 
For a sequence in $\mathcal{A C}_{n}$ approaching $\left(d_{0}, \ldots, d_{n-1}\right) \in \mathcal{H I}_{n}$, where $d_{i_{0}}=d_{i_{0}+1}=\infty$, an arbitrary element is the side length collection of a cyclic polygon $\left\{x_{0}, \ldots, x_{n-1}\right\}$ with longest sides containing $x_{i_{0}}$. We divide the convex hull of $\left\{x_{0}, \ldots, x_{n-1}\right\}$ into a non-overlapping union of cyclic triangles using diagonals from $x_{i_{0}}$, with side lengths given by the diagonal functions $\ell_{i_{0}, j}$ of Corollary 1.21, for $j \neq i_{0}, i_{0}+1$. We compute values of $D_{0}$ by summing the areas of these triangles. Since they are cyclic, their side lengths satisfy the hypotheses of the claim.

The resulting extensions of $D_{0}$ are clearly symmetric and continuous. For $\left(d_{0}, \ldots, d_{n-1}\right)$ and $\left(d_{0}^{\prime}, \ldots, d_{n-1}^{\prime}\right) \in \mathcal{H C}_{n}$ such that $d_{i} \leq d_{i}^{\prime}$ for each $i$, if $d_{i_{0}}$ is maximal among the $d_{i}$ and $d_{i_{1}}^{\prime}$ is maximal among the $d_{i}^{\prime}$, then

$$
d_{i_{1}}^{\prime}>d_{i_{0}}^{\prime} \geq d_{i_{0}} \quad \text { and } \quad d_{i_{0}}^{\prime} \geq d_{i_{0}}>d_{i_{1}} .
$$

Thus, exchanging $d_{i_{1}}^{\prime}$ with $d_{i_{0}}^{\prime}$ does not change the fact that $d_{i} \leq d_{i}^{\prime}$ for all $i$. Having made the exchange, and assuming without loss of generality that $i_{0}=0$, some calculus shows that $D_{0}$ increases along the graph of the straight-line path from $\left(d_{1}, \ldots, d_{n-1}\right)$ to $\left(d_{1}^{\prime}, \ldots, d_{n-1}^{\prime}\right)$ in graph $\left(h_{0}\right)$ (recall Corollary 3.5$)$.

The corresponding monotonicity property of $D_{0}$ on $\mathcal{H} \mathcal{I}_{n}$ follows by direct comparison. For $\left(d_{0}, \ldots, d_{n-1}\right) \in \mathcal{H C}_{n}$ and $\left(d_{0}^{\prime}, \ldots, d_{n-1}^{\prime}\right) \in$ $\mathcal{H} \mathcal{I}_{n}$, we note that, if $d_{i} \leq d_{i}^{\prime}$ for all $i$, then for $i_{0}$ such that $d_{i_{0}}$ is maximal among the $d_{i}, d_{i_{0}}^{\prime}=\infty$.

Proposition 3.9. Let $C$ be a horocycle with ideal point $v$, and for some $n \geq 3$, suppose $\left\{x_{0}, \ldots, x_{n-1}\right\} \subset C \cup\{v\}$ is a horocyclic ideal $n$-gon with ideal vertex $x_{i_{0}}=v$. For each $i>0$, there is a unique half-space $\mathcal{H}_{i}$ bounded by the geodesic through $x_{i}$ and $x_{i-1}$ such that $x_{j} \in \overline{\mathcal{H}}_{i}$ for all $j$, where $\overline{\mathcal{H}}_{i}$ is the closure of $\mathcal{H}_{i}$ in $\mathbb{C} \cup\{\infty\}$. Similarly, there is a unique half-space $\mathcal{H}_{0}$ bounded by the geodesic through $x_{0}$ and $x_{n-1}$ such that $x_{j} \in \overline{\mathcal{H}}_{0}$ for all $j$.

Say

$$
P=\bigcap_{i=0}^{n-1} \mathcal{H}_{i}
$$

is the convex hull of $\left\{x_{0}, \ldots, x_{n-1}\right\}$. It is a convex polygon contained in the horoball bounded by $C$, with vertex set $\left\{x_{0}, \ldots, \widehat{x}_{i_{0}}, \ldots, x_{n-1}\right\}$ and edge set $\left\{\gamma_{i}\right\}$, where $\gamma_{i}$ is the geodesic joining $x_{i}$ to $x_{i-1}$ for each $i$. 
$P$ decomposes as the non-overlapping union

$$
\bigcup_{\substack{i \neq i_{0} \\ i_{0}+1}} T_{i}
$$

where $T_{i}$ is the convex hull of $x_{i}, x_{i-1}$ and $v$ for each such $i$.

Now suppose $\left\{x_{0}, \ldots, x_{n-1}\right\} \subset C$ is a horocyclic $n$-gon with maximal side length $d_{i_{0}}=\operatorname{dist}\left(x_{i_{0}-1}, x_{i_{0}}\right)$. It is the vertex set of its convex hull, a compact, convex polygon, $P$, contained in the horoball bounded by $C$. The edges of $P$ are the geodesic arcs $\gamma_{i}$ joining $x_{i-1}$ to $x_{i}$ for each $i>0$, together with $\gamma_{0}$ joining $x_{n-1}$ to $x_{0}$. Taking $T_{i}$ as in the previous case for each $i$,

$$
P \cup T_{i_{0}}=\bigcup_{i \neq i_{0}} T_{i},
$$

is a convex polygon.

In each case above, the area of $P$ is given by $D_{0}\left(d_{0}, \ldots, d_{n-1}\right)$ from Proposition 3.7, where $d_{i}=\operatorname{dist}\left(x_{i}, x_{i-1}\right)$ for each $i$ (in particular, $d_{i}=\infty$ if $x_{i}$ or $x_{i-1}$ is $\left.v\right)$.

Proof. The geodesics of the upper half-plane $\mathbb{H}^{2}$ are the intersections with $\mathbb{H}^{2}$ of vertical Euclidean straight lines and Euclidean circles centered in $\mathbb{R}$. It follows from this description that any two distinct points of $\mathbb{H}^{2}$ are contained in the closure in $\mathbb{C} \cup\{\infty\}$ of a unique geodesic. (In particular, the closure of a vertical Euclidean straight line contains $\infty$.)

Now taking $C=C_{\infty}$, we address the case that $\left\{x_{0}, \ldots, x_{n-1}\right\}$ is a horocyclic ideal polyhedron. If $x_{i_{0}}=\infty$, then, in order of increasing real parts,

$$
x_{i_{0}+1}, \ldots, x_{n-1}, x_{0}, \ldots, x_{i_{0}-1},
$$

lie on $C_{\infty}$. The half-space $\mathcal{H}_{i_{0}+1}$, described above is therefore the region to the right of the vertical Euclidean straight line through $x_{i_{0}+1}$, and $\mathcal{H}_{i_{0}}$ is to the left of the vertical line through $x_{i_{0}-1}$. For each $i \neq i_{0}$ or $i_{0}+1, \mathcal{H}_{i}$ is the region outside the Euclidean circle through $x_{i}$ and $x_{i-1}\left(x_{n-1}\right.$ if $\left.i=0\right)$ that is centered in $\mathbb{R}$.

It is now easy to see that the edges of $P=\bigcap \mathcal{H}_{i}$ are the $\gamma_{i}$ described above, and the vertices consist of all $x_{i}$ except $x_{i_{0}}$ (compare the proof of Lemma 2.1). $P$ is divided into the $T_{i}, i \neq i_{0}, i_{0}+1$, by the vertical 
lines through the $x_{i}, i \neq i_{0}, i_{0} \pm 1$. This is because $T_{i}$ is bounded by the vertical lines through $x_{i}$ and $x_{i-1}$, and the circular arc containing both of these points, for each $i$.

If $\left\{x_{0}, \ldots, x_{n-1}\right\}$ is horocyclic but not horocyclic ideal, then the proof that it is the vertex set of its convex hull $P$ follows that of Lemma 2.1, along with the fact that $P$ is compact and the description of the edge set. Again, taking $C=C_{\infty}$, the key difference between this case and the previous one is that, since all $x_{i}$ lie on $C_{\infty}$, all edges are compact circular arcs. In particular, if $d_{i_{0}}$ is maximal among the $d_{i}$, then $x_{i_{0}}$ has minimal real coordinate, $x_{i_{0}-1}$ has maximal real coordinate, and $\mathcal{H}_{i_{0}}$ is the region inside the Euclidean circle through $x_{i_{0}}$ and $x_{i_{0}-1}$ that is centered in $\mathbb{R}$.

Given this fact, we note that $P \cup T_{i_{0}}$ is the convex hull of

$$
\left\{x_{0}, \ldots, x_{i_{0}-1}, v, x_{i_{0}}, \ldots, x_{n-1}\right\},
$$

a horocyclic ideal $(n+1)$-gon, so its decomposition follows from the previous case.

That the area of either $P$ above is $D_{0}\left(d_{0}, \ldots, d_{n-1}\right)$ follows from their decompositions and the fact that $T_{i}$ has area $\pi-2 \sin ^{-1}\left(1 / \cosh \left(d_{i} / 2\right)\right)$ for each $i$. This in turn follows again from the Gauss-Bonnet formula for the area of hyperbolic triangles, see [10, Theorem 3.5.5]. $T_{i}$ is a "generalized hyperbolic triangle" in the terminology of [10, subsection $3.5]$, with ideal vertex $v$, so its angle at $v$ is defined to be 0 . An exercise in Euclidean geometry (recalling from the proof of Proposition 3.4 that $\sinh \left(d_{i} / 2\right)=\left|x_{i}-x_{i-1}\right| / 2$, and noting that the hyperbolic and Euclidean metrics on $\mathbb{H}^{2}$ are conformal; see Definition 1.1) establishes that its angles at $x_{i}$ and $x_{i-1}$ are each $\alpha$ satisfying $\sin \alpha=1 / \cosh \left(d_{i} / 2\right)$. (Compare [14, 2.6.12].)

4. ....and beyond! To this point, we have proved that a tuple $\left(d_{0}, \ldots, d_{n-1}\right) \in(0, \infty)^{n}$ is the side length collection of a unique cyclic or horocyclic $n$-gon in $\mathbb{H}^{2}$ if and only if $\sinh \left(d_{i} / 2\right) \leq \sum_{j \neq i} \sinh \left(d_{j} / 2\right)$, for all $i$. This condition implies that $d_{i}<\sum_{j \neq i} d_{j}$, since the hyperbolic sine has positive first and second derivatives on $(0, \infty)$.

Here, we address the remaining case: any $\left(d_{0}, \ldots, d_{n-1}\right) \in(0, \infty)^{n}$ such that $\sinh \left(d_{i} / 2\right)>\sum_{j \neq i} \sinh \left(d_{j} / 2\right)$, but $d_{i} \leq \sum_{j \neq i} d_{j}$, for some 
$i$ is the side length collection of an equidistant polygon, with vertices equidistant from a fixed geodesic.

In the upper half-plane model, such an equidistant locus is the intersection with $\mathbb{H}^{2}$ of a circle in $\mathbb{C}$ not entirely contained in $\overline{\mathbb{H}}^{2}$. It is literally beyond infinity!

Definition 4.1. For $J \geq 0$, the $J$-equidistant locus to a geodesic $\gamma$ in $\mathbb{H}^{2}$ is the collection of points that have distance $J$ from $\gamma$. For $n \geq 3$, a collection $\left\{x_{0}, \ldots, x_{n-1}\right\}$ of distinct points on a component $C$ of the $J$-equidistant locus to $\gamma$ is an equidistant $n$-gon if, for some $i_{0}$, the collection $\left\{x_{i_{0}}, \ldots, x_{n-1}, x_{0}, \ldots, x_{i_{0}-1}\right\}$ is linearly ordered on $C$. In the orientation that $C$ inherits as a boundary component of the region it bounds with $\gamma$, the compact arc of $C$ is bounded by $x_{i}$ and $x_{i-1}$ points from $x_{i-1}$ to $x_{i}$ for each $i \neq i_{0}$. Here, we take $0-1$ to be $n-1$. We say $x_{i_{0}}$ is first among the $x_{i}$.

The collar radius of $\left\{x_{0}, \ldots, x_{n-1}\right\}$ is the distance from the $x_{i}$ to $\gamma$. Its side length collection is $\left(d_{0}, \ldots, d_{n-1}\right) \in(0, \infty)^{n}$, where $d_{i}=\operatorname{dist}\left(x_{i-1}, x_{i}\right)$ for $i>0$ and $d_{0}=\operatorname{dist}\left(x_{n-1}, x_{0}\right)$.

We prove existence and uniqueness of equidistant $n$-gons by a strategy parallel to the one for cyclic $n$-gons. The role of the circle center is played here by the geodesic $\gamma$, and the angle from $x$ to $y$ by the distance from the orthogonal projection of $x$ to the projection of $y$.

Lemma 4.2. If points $x$ and $y$ in $\mathbb{H}^{2}$ with $\operatorname{dist}(x, y)=d \geq 0$ each have distance $J \geq 0$ from a hyperbolic geodesic $\gamma$, then the orthogonal projections of $x$ and $y$ to $\gamma$ are at distance:

$$
\psi(d, J)=2 \sinh ^{-1}(\sinh (d / 2) / \cosh J) .
$$

This is a continuous function on $[0, \infty)^{2}$, smooth in its interior. For fixed $d>0, \psi(d, J)$ decreases in $J$ on $[0, \infty)$ with $\psi(d, 0)=d$, $\lim _{J \rightarrow \infty} \psi(d, J)=0$, and the partial derivative $(\partial / \partial J) \psi(d, J)$ is given by:

$$
\frac{-2 \sinh (d / 2) \sinh J}{\cosh J \sqrt{\cosh ^{2} J+\sinh ^{2}(d / 2)}}=-2 \tanh J \tanh (\psi(d, J) / 2) .
$$


Proof. Let $p$ and $q$ be the orthogonal projections of $x$ and $y$ to $\gamma$, respectively. The arc joining $x$ to $p$ meets $\gamma$ perpendicularly (hence the term orthogonal projection), as does the arc joining $y$ to $q$, so these arcs and hence also $x$ and $y$ are exchanged by reflection in the perpendicular bisector of the arc of $\gamma$ joining $p$ to $q$. If $Q$ is the quadrilateral with vertices at $x, y, p$, and $q$, this perpendicular bisector thus divides $Q$ into isometric quadrilaterals $Q_{0}$ and $Q_{1}$ with three right angles each.

Suppose $Q_{0}$ contains $x$. If $\alpha$ is its angle at $x$, then hyperbolic trigonometry gives the following relations between $\alpha$ and the side lengths:

$$
\begin{aligned}
\cos \alpha & =\sinh h \sinh (\psi(d, J) / 2) \\
\cosh (d / 2) & =\frac{\cosh (\psi(d, J) / 2)}{\sin \alpha} \\
\cosh J & =\frac{\cosh h}{\sin \alpha} .
\end{aligned}
$$

Here $h$ is the length of the side of $Q_{0}$ that lies in the perpendicular bisector of the geodesic from $x$ to $y$. Equation 4.1 follows from [10, Theorem 3.5.10] and the other two from [10, Theorem 3.5.7]. Some manipulations give the formula for $\psi(d, J)$, and also:

$$
\sin \alpha=\frac{\sqrt{\cosh ^{2} J+\sinh ^{2}(d / 2)}}{\cosh J \cosh (d / 2)} .
$$

The remaining assertions are straightforward.

Lemma 4.3. If $\left\{x_{0}, \ldots, x_{n-1}\right\}$ is an equidistant $n$-gon with collar radius $J$ and side length collection $\left(d_{0}, \ldots, d_{n-1}\right)$, such that $x_{i_{0}}$ is first among the $x_{i}$, then

$$
\begin{aligned}
d_{i_{0}} & =L\left(J, d_{0}, \ldots, \widehat{d_{i_{0}}}, \ldots, d_{n-1}\right) \\
& \doteq 2 \sinh ^{-1}\left[\cosh J \sinh \left(\frac{1}{2} \sum_{i \neq i_{0}} \psi\left(d_{i}, J\right)\right)\right] .
\end{aligned}
$$

Moreover, given any fixed collection of $d_{i}>0$, for $i \neq i_{0}$, and $J \geq 0$, there is an equidistant $n$-gon $\left\{x_{0}, \ldots, x_{n-1}\right\}$ satisfying the hypotheses above, and any two such are isometric.

Proof. Given $d_{i}>0$ for $i \neq i_{0}$, and $J \geq 0$, fix a hyperbolic geodesic $\gamma$, a component $C$ of the $J$-equidistant locus to $\gamma$, and $x_{i_{0}} \in C$. Let 
$p: \mathbb{H}^{2} \rightarrow \gamma$ be the orthogonal projection, and for each $y \in \gamma$, let $p^{-1}(y)$ be the unique intersection point of $C$ with the preimage of $y$ under $p$.

Let $y_{i_{0}}=p\left(x_{i_{0}}\right)$. We will recursively produce the $x_{i}$ for $i \neq i_{0}$, starting at $i=i_{0}+1$ and taking $i$ modulo $n$. Let $y_{i}$ be the point on $\gamma$ at distance $\psi\left(d_{i}, J\right)$ from $y_{i-1}(\bmod n)$ with the property that the compact arc bounded by $y_{i}$ and $y_{i-1}$ points toward $y_{i-1}$ in the boundary orientation that $\gamma$ inherits from the region it bounds with $C$. Note that the restriction of $p$ to $C \rightarrow \gamma$ reverses this orientation, so taking $x_{i}=p^{-1}\left(y_{i}\right)$ for each $i$ we obtain a cyclic $n$-gon on $C$ with $x_{i_{0}}$ first among the $x_{i}$.

Lemma 4.2 implies that $\operatorname{dist}\left(x_{i}, x_{i-1}\right)=d_{i}$ for each $i \neq i_{0}$. By construction, the distance from $y_{i_{0}}$ to $y_{i_{0}-1}$ is $\sum_{i \neq i_{0}} \psi\left(d_{i}, J\right)$, so another application of Lemma 4.2 implies that $\operatorname{dist}\left(x_{i_{0}}, x_{i_{0}-1}\right)$ satisfies the formula for $d_{i_{0}}$ described above. It follows that $\left\{x_{0}, \ldots, x_{n-1}\right\}$ is an equidistant $n$-gon with side length collection $\left(d_{0}, \ldots, d_{n-1}\right)$. By construction, it has collar radius $J$ and $x_{i_{0}}$ first among the $x_{i}$.

Another equidistant $n$-gon with the properties of $\left\{x_{0}, \ldots, x_{n-1}\right\}$, on a component of the equidistant locus to a hyperbolic geodesic $\gamma^{\prime}$, can be taken to $\left\{x_{0}, \ldots, x_{n-1}\right\}$ by the following sequence of isometries: take $\gamma^{\prime}$ to $\gamma$, apply an order-2 rotation around a point of $\gamma$ if necessary (to exchange components of the $J$-equidistant locus), then translate in $\gamma$.

Lemma 4.4. For any $n \geq 3$, the function $L$, defined in Lemma 4.3, is continuous on $[0, \infty)^{n}$ and smooth on $(0, \infty)^{n}$. Fixing $\left(d_{1}, \ldots, d_{n-1}\right)$ and taking the restriction of $L$ to $[0, \infty) \times\left\{\left(d_{1}, \ldots, d_{n-1}\right)\right\}$ as a function of $J$, we have

$$
\begin{aligned}
\frac{\partial}{\partial J} L(J) & <0 \quad \text { for all } J>0, \\
L(0) & =\sum_{i=1}^{n-1} d_{i}
\end{aligned}
$$

and

$$
\lim _{J \rightarrow \infty} L(J)=2 \sinh ^{-1}\left(\sum_{i=1}^{n-1} \sinh \left(d_{i} / 2\right)\right) .
$$


Proof. The value of $L(0)$ follows directly from its definition, as does the computation below:

$$
\begin{aligned}
\frac{1}{2} \cosh (L(J) / 2) \frac{\partial L}{\partial J}= & \sinh J\left[\sinh \left(\frac{1}{2} \sum_{i=1}^{n-1} \psi\left(d_{i}, J\right)\right)\right. \\
& \left.-\cosh \left(\frac{1}{2} \sum_{i=1}^{n-1} \psi\left(d_{i}, J\right)\right) \sum_{i=1}^{n-1} \tanh \left(\psi\left(d_{i}, J\right) / 2\right)\right] .
\end{aligned}
$$

We also appealed to Lemma 4.2 for the derivative of $\psi$ with respect to $J$. That $\partial L / \partial J<0$ now follows from the fact that $\sum \tanh x_{i}>$ $\tanh \left(\sum x_{i}\right)$. This follows by induction along the lines of Claim 1.9 in the proof of Proposition 1.6, using the angle addition identity for hyperbolic tangent:

$$
\tanh (x+y)=\frac{\tanh x+\tanh y}{1+\tanh x \tanh y} .
$$

For the limit $J \rightarrow \infty$, we note that the quantity in brackets in the definition of $L$ is of the form $\infty \cdot 0$, so rewriting $\cosh J$ as $1 /(1 / \cosh J)$, applying l'Hôpital's rule and simplifying, gives:

$$
\begin{array}{r}
\lim _{J \rightarrow \infty} \cosh J \sinh \left(\frac{1}{2} \sum_{i=1}^{n-1} \psi\left(d_{i}, J\right)\right)=\lim _{J \rightarrow \infty} \cosh \left(\frac{1}{2} \sum_{i=1}^{n-1} \psi\left(d_{i}, J\right)\right) \\
\sum_{i=1}^{n-1} \frac{\sinh \left(d_{i} / 2\right) \cosh J}{\sqrt{\cosh ^{2} J+\sinh ^{2}\left(d_{i} / 2\right)}}=\sum_{i=1}^{n-1} \sinh \left(d_{i} / 2\right) .
\end{array}
$$

It follows that the limit of $L$ is as described.

Proposition 4.5. If $\left(d_{0}, \ldots, d_{n-1}\right)$ is the side length collection of an equidistant $n$-gon with $d_{i_{0}}$ maximal among the $d_{i}$, then $d_{i_{0}} \leq \sum_{i \neq i_{0}} d_{i}$, but $\sinh \left(d_{i_{0}} / 2\right)>\sum_{i \neq i_{0}} \sinh \left(d_{i} / 2\right)$. For $n \geq 3$, any $\left(d_{0}, \ldots, d_{n-1}\right) \in$ $(0, \infty)^{n}$ satisfying the above inequalities is the side length collection of a unique equidistant $n$-gon, up to isometry. In particular, the collar radius is uniquely determined by, and moreover, a symmetric function of, $\left(d_{0}, \ldots, d_{n-1}\right)$. Two equidistant $n$-gons are isometric if and only if their side length collections differ by a cyclic permutation. 
Proof. For an equidistant polygon $\left\{x_{0}, \ldots, x_{n-1}\right\}$, with side length collection $\left(d_{0}, \ldots, d_{n-1}\right)$ and collar radius $J$, if $x_{i_{0}}$ is first among the $x_{i}$, then Lemma 4.3 implies that

$$
d_{i_{0}}=L_{i_{0}}(J) \doteq 2 \sinh ^{-1}\left[\cosh J \sinh \left(\frac{1}{2} \sum_{i \neq i_{0}} \psi\left(d_{i}, J\right)\right)\right] .
$$

By Lemma 4.4, $L_{i_{0}}$ is continuous and strictly decreasing on $[0, \infty)$, with $L_{i_{0}}(J) \leq \sum_{i \neq i_{0}} d_{i}$ and $\sinh \left(L_{i_{0}}(J) / 2\right)>\sum_{i \neq i_{0}} \sinh \left(d_{i} / 2\right)$, for all $J$. The same inequalities therefore hold for $d_{i_{0}}$, which in particular is maximal among the $d_{i}$.

On the other hand, given $\left(d_{0}, \ldots, d_{n-1}\right)$, such that the maximal entry $d_{i_{0}}$ satisfies $d_{i_{0}} \leq \sum_{i \neq i_{0}} d_{i}$ and $\sinh \left(d_{i_{0}} / 2\right)>\sum_{i \neq i_{0}} \sinh \left(d_{i} / 2\right)$, since $L_{i_{0}}$ as defined above is continuous on $[0, \infty)$, there exists some $J \geq 0$ such that $d_{i_{0}}=L_{i_{0}}(J)$. Therefore, by Lemma 4.3 , there is an equidistant $n$-gon $B$ with side length collection $\left(d_{0}, \ldots, d_{n-1}\right)$ and collar radius $J$. Moreover, since $L_{i_{0}}$ is strictly decreasing, this $J$ is uniquely determined by $\left(d_{0}, \ldots, d_{n-1}\right)$. Therefore, by Lemma 4.3 again, $P$ is unique up to isometry.

We note that cyclically relabeling the vertices of an equidistant polygon produces an isometric (by the identity map) equidistant polygon, whose side length collection is obtained from the original by the same cyclic relabeling. On the other hand, it is clear that an isometry of equidistant polygons takes the side length collection of one to a cyclic permutation of the side length collection of the other.

We note that $L(J)$ is symmetric in $\left(d_{1}, \ldots, d_{n-1}\right)$. This is clear by inspecting its definition in Lemma 4.4. Since the collar radius $J\left(d_{0}, \ldots, d_{n-1}\right)$ of an equidistant $n$-gon with side length collection $\left(d_{0}, \ldots, d_{n-1}\right)$ is determined by the equation,

$$
d_{0}=L\left(J, d_{0}, \ldots, \widehat{d}_{i_{0}}, \ldots, d_{n-1}\right),
$$

it is therefore invariant under any permutation of the entries fixing the $i_{0}$ place. But we have already shown that cyclically permuting side lengths yields isometric equidistant polygons, thus with identical collar radii. Symmetricity of collar radius follows.

The following corollary is immediate. Here we use marked as in Corollary 1.16 . 
Corollary 4.6. For $n \geq 3$, marked equidistant $n$-gons in $\mathbb{H}^{2}$ are parametrized up to isometry by:

$\mathcal{E}_{n}=\left\{\mathbf{d} \mid \sinh \left(d_{i_{0}} / 2\right)>\sum_{i \neq i_{0}} \sinh \left(d_{i} / 2\right)\right.$ but $d_{i_{0}} \leq \sum_{i \neq i_{0}} d_{i}$, for some $\left.i_{0}\right\}$.

Above, we take $\mathbf{d}=\left(d_{0}, \ldots, d_{n}\right) \in\left(\mathbb{R}^{+}\right)^{n}$. The topological frontier of $\mathcal{E}_{n}$ in $(0, \infty)^{n}$ is

$$
\mathcal{H C}_{n} \sqcup\left\{\left(d_{0}, \ldots, d_{n-1}\right) \mid d_{i}=\sum_{j \neq i} d_{j} \quad \text { for some } i\right\} .
$$

Proposition 4.7. The function $J: \mathcal{E}_{n} \rightarrow \mathbb{R}^{n}$ that records the collar radius of equidistant $n$-gons is symmetric and continuous on $\mathcal{E}_{n}$ and smooth on its interior. If $d_{i_{0}}$ is maximal among the $d_{i}$, then:

$$
\frac{\partial J}{\partial d_{i}}(\mathbf{d})=\frac{1}{2} \frac{ \pm \cosh \left(d_{i} / 2\right) \operatorname{coth} J \sqrt{\frac{\cosh ^{2} J+\sinh ^{2}\left(d_{i_{0}} / 2\right)}{\cosh ^{2} J+\sinh ^{2}\left(d_{i} / 2\right)}}}{\sinh \left(d_{i_{0}} / 2\right)-\sum_{j \neq i_{0}} \sinh \left(d_{j} / 2\right) \sqrt{\frac{\cosh ^{2} J+\sinh ^{2}\left(d_{i_{0}} / 2\right)}{\cosh ^{2} J+\sinh ^{2}\left(d_{j} / 2\right)}}} .
$$

Here the $\pm i s+$ for $i=i_{0}$ and - otherwise. Values of $J$ approach infinity on sequences approaching $\mathcal{H C}_{n}$, and $J\left(d_{0}, \ldots, d_{n-1}\right)=0$, if and only if $d_{i_{0}}=\sum_{i \neq i_{0}} d_{i}$ for some $i$.

Proof. Collar radius $J$ satisfies the equation $d_{i_{0}}=L\left(J, d_{0}, \ldots, \widehat{d}_{i_{0}}\right.$, $\left.\ldots, d_{n-1}\right)$, by Lemma 4.3. Applying Lemma 4.4 and the implicit function theorem, we find that $J$ is smooth on $\mathcal{E}_{n}$, with:

$$
\begin{aligned}
\frac{\partial J}{\partial d_{i_{0}}} & =\frac{1}{\partial L / \partial J} \\
\frac{\partial J}{\partial d_{i}} & =-\frac{\partial L / \partial d_{i}}{\partial L / \partial J}, \quad i \neq i_{0},
\end{aligned}
$$

compare the proof of Lemma 1.19. Keeping in mind that $d_{i_{0}}=L$ at 
the point in question, the computation from (4.3) yields:

$$
\begin{aligned}
\frac{\partial L}{\partial J}= & 2 \frac{\sinh J \sqrt{\cosh ^{2} J+\sinh ^{2}\left(d_{i_{0}} / 2\right)}}{\cosh J \cosh \left(d_{i_{0}} / 2\right)} \\
& {\left[\frac{\sinh \left(d_{i_{0}} / 2\right)}{\sqrt{\cosh ^{2} J+\sinh ^{2}\left(d_{i_{0}} / 2\right)}}-\sum_{i \neq i_{0}} \frac{\sinh \left(d_{i} / 2\right)}{\sqrt{\cosh ^{2} J+\sinh ^{2}\left(d_{i} / 2\right)}}\right] . }
\end{aligned}
$$

Another computation gives:

$$
\frac{\partial L}{\partial d_{i}}=\frac{\cosh \left(d_{i} / 2\right)}{\cosh \left(d_{i_{0}} / 2\right)} \sqrt{\frac{\cosh ^{2} J+\sinh ^{2}\left(d_{i_{0}} / 2\right)}{\cosh ^{2} J+\sinh ^{2}\left(d_{i} / 2\right)}} .
$$

The derivative computation described above follows. That $J$ takes the value 0 at $\left(d_{0}, \ldots, d_{n-1}\right)$, if and only if, $d_{i_{0}}=\sum_{i \neq i_{0}} d_{i_{0}}$ for some $i_{0}$, follows from Lemma 4.4, since $L$ satisfies $L(0)=\sum_{i \neq i_{0}} d_{i}$ and $\partial / \partial J L<0$, on $(0, \infty)$, with $d_{i_{0}}=L\left(J, d_{0}, \ldots, \widehat{d}_{i_{0}}, \ldots, d_{n-1}\right)$ by Lemma 4.3.

The argument that $J \rightarrow \infty$ approaches $\mathcal{H C}_{n}$ is essentially identical to the proof of Lemma 3.6. It again uses Lemma 4.4, since

$$
\lim _{J \rightarrow \infty} L(J)=2 \sinh ^{-1}\left(\sum_{i \neq i_{0}} \sinh \left(d_{i} / 2\right)\right) .
$$

Proposition 4.8. For $n \geq 3$, an equidistant $n$-gon $\left\{x_{0}, \ldots, x_{n-1}\right\}$ in a component, $C$, of the $J$-equidistant locus to a geodesic $\gamma$ in $\mathbb{H}^{2}$, is the vertex set of its convex hull, a compact, convex polygon, $P$, contained region between $C$ and $\gamma$. The edges of $P$ are the geodesic arcs $\gamma_{i}$ joining $x_{i-1}$ to $x_{i}$ for each $i>0$, together with $\gamma_{0}$ joining $x_{n-1}$ to $x_{0}$.

For each $i$, let $Q_{i}$ be the quadrilateral with vertices at $x_{i-1}, x_{i}$ (if $i=0$, at $x_{0}$ and $\left.x_{n-1}\right)$ and their projections to $\gamma$. Then, if $x_{i_{0}}$ is first among the $x_{i}, P \cup Q_{i_{0}}$ decomposes as the non-overlapping union $\bigcup_{i \neq i_{0}} Q_{i}$.

Proof. The description of $P$ follows as in Lemma 2.1. The key fact is that, for any $x$ and $y$ on $C$, the geodesic through $x$ and $y$ intersects $C$ in $\{x, y\}$; and it intersects the region bounded by $C$ and $\gamma$ in the geodesic $\operatorname{arc}$ joining $x$ to $y$. In particular, this region is convex. This in turn 
follows from convexity of the hyperbolic metric (see e.g., the discussion at the beginning of [2, Chapter II.2], in particular, Proposition 2.2 there).

The decomposition of $P \cup Q_{i_{0}}$ follows similarly to the non-centered case of Proposition 2.2.

Proposition 4.9. The function $D_{0}: \mathcal{E}_{n} \rightarrow \mathbb{R}^{+}$that records area of equidistant $n$-gons is symmetric and continuous on $\mathcal{E}_{n}$ and smooth on its interior. It satisfies:

$$
\frac{\partial D_{0}}{\partial d_{i}}= \begin{cases}-\sqrt{\left(1 / \sinh ^{2} J\right)+\left(1 / \cosh ^{2}\left(d_{i_{0}} / 2\right)\right)} & i=i_{0}, \\ \sqrt{\left(1 / \sinh ^{2} J\right)+\left(1 / \cosh ^{2}\left(d_{i} / 2\right)\right)} & \text { otherwise, }\end{cases}
$$

where $d_{i_{0}}$ is maximal among the $d_{i} . D_{0}\left(d_{0}, \ldots, d_{n-1}\right)=0$ if and only if $d_{i_{0}}=\sum_{i \neq i_{0}} d_{i}$ for some $i_{0}$, and $D_{0}$ extends continuously to $\mathcal{H C}_{n}$ by the formula of Proposition 3.7.

Proof. The proof follows our strategy from the cyclic case. Let $\left\{x_{0}, \ldots, x_{n-1}\right\}$ be an equidistant polygon with side length collection $\left(d_{0}, \ldots, d_{n-1}\right)$, and suppose $x_{i_{0}}$ is first among the $x_{i}$. For each $i$, let $Q_{i}$ be the quadrilateral defined in Proposition 4.8. As we observed in the proof of Lemma 4.2, $Q_{i}$ admits a reflection exchanging $x_{i-1}$ with $x_{i}$, so it has identical angles there. Call this angle $\alpha_{i}$. It is determined by formula (4.2) with $d_{i}$ substituted for $d$. Note that $\alpha_{i}$ is continuous in $d$ and $J$ on $[0, \infty)^{2}$ and smooth on $(0, \infty)^{2}$. Since $Q_{i}$ has right angles at its vertices on $\gamma$, it has area $\pi-2 \alpha_{i}$.

Assuming that $J$ depends on $d$, a computation gives:

$$
\frac{\partial}{\partial d_{i}} \operatorname{area}\left(Q_{i}\right)=\frac{\left(\sinh J / \cosh \left(d_{i} / 2\right)\right)+2\left(\sinh \left(d_{i} / 2\right) / \cosh J\right)\left(\partial J / \partial d_{i}\right)}{\sqrt{\cosh ^{2} J+\sinh ^{2}\left(d_{i} / 2\right)}} .
$$

For $j \neq i$, the derivative of $Q_{i}$ with respect to $d_{j}$ is identical to the above except that the numerator lacks the term $\sinh J / \cosh \left(d_{i} / 2\right)$, and there $\partial J / \partial d_{j}$ replaces $\partial J / \partial d_{i}$. Here $J=J\left(d_{0}, \ldots, d_{n-1}\right)$ is the collar radius of $\left\{x_{0}, \ldots, x_{n-1}\right\}$. 
The decomposition of Proposition 4.8 gives

$$
D_{0}\left(d_{0}, \ldots, d_{n-1}\right)=\left(\sum_{i \neq i_{0}} \operatorname{area}\left(Q_{i}\right)\right)-\operatorname{area}\left(Q_{i_{0}}\right) .
$$

By Proposition $4.7, J\left(d_{0}, \ldots, d_{n-1}\right)=0$ if and only if $d_{i_{0}}=\sum_{i \neq i_{0}} d_{i}$ for some $i_{0}$, so it follows from that result and properties of area $\left(Q_{i}\right)$ described above that $D_{0}$ is smooth on the interior of $\mathcal{E}_{n}$. Taking derivatives now yields:

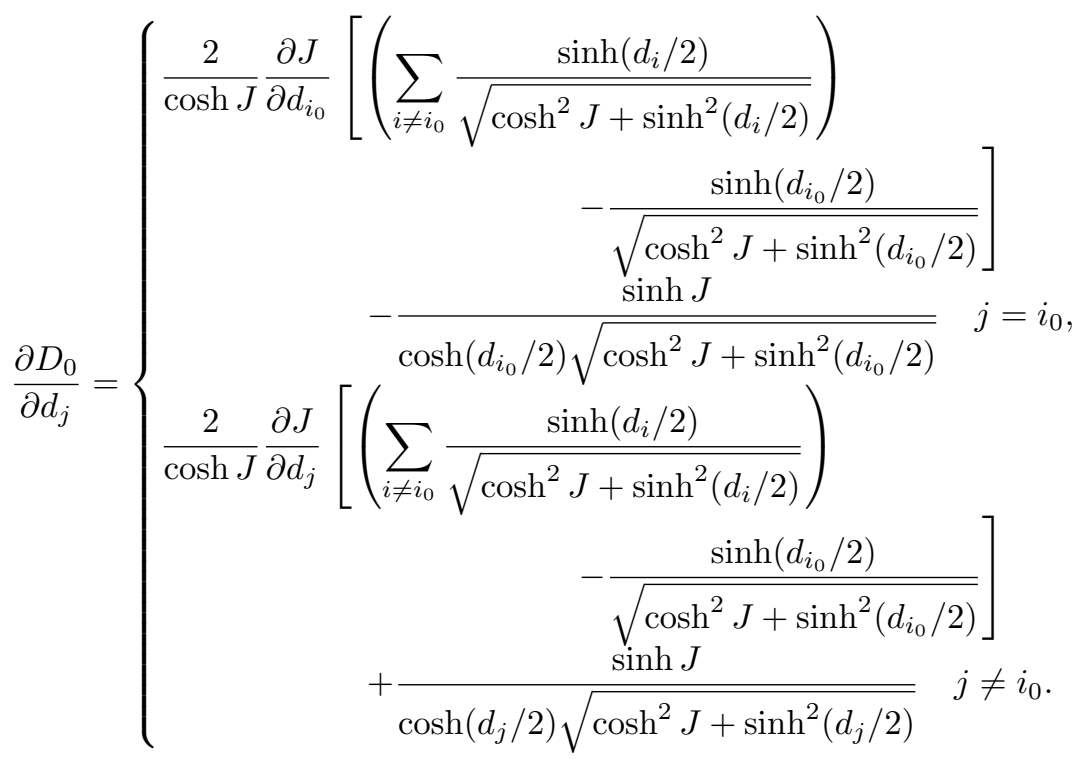

Substituting for $\partial J / \partial d_{j}$ and simplifying yields the formulas claimed, noting in particular that $\cosh ^{2}\left(d_{j} / 2\right)+\sinh ^{2} J=\sinh ^{2}\left(d_{j} / 2\right)+\cosh ^{2} J$ by trading a sum with one from one term to the other.

If $\left(d_{0}, \ldots, d_{n-1}\right) \in \mathcal{E}_{n}$ has $d_{i_{0}}=\sum_{i \neq i_{0}} d_{i}$ for some $i$, then, by Proposition $4.7, J\left(d_{0}, \ldots, d_{n-1}\right)=0$, so for each $i, \alpha_{i}=\pi / 2$ by formula (4.2). It follows that $Q_{i}$ as described in Proposition 4.8 has area 0 , so by that result $D_{0}\left(d_{0}, \ldots, d_{n-1}\right)=0$ as well. By the results above, $D_{0}$ is strictly decreasing along the ray, $\left(d_{0}, \ldots, d_{i_{0}-1}\right) \times\left(h_{0}, \sum_{i \neq i_{0}} d_{i}\right] \times$ $\left(d_{i_{0}+1}, \ldots, d_{n-1}\right)$, so it takes the value 0 only where $d_{i_{0}}=\sum_{i \neq i_{0}} d_{i}$. 
Finally, since values of $J$ limit to infinity on sequences approaching $\mathcal{H C}_{n}$ by Proposition 4.7, values of the $\alpha_{i}$ determined by (4.2) limit to $\sin ^{-1}\left(\tanh \left(d_{i} / 2\right)\right)=\cos ^{-1}\left(1 / \cosh \left(d_{i} / 2\right)\right)$.

Corollary 4.10. For $n \geq 3$, $\mathbf{d}=\left(d_{1}, \ldots, d_{n-1}\right) \in\left(\mathbb{R}^{+}\right)^{n-1}$, and $d_{0} \geq D=\max \left\{d_{i}\right\},\left(d_{0}, \mathbf{d}\right)$ lies in:

$$
\begin{cases}\mathcal{C}_{n} & \text { if } D \leq d_{0}<b_{0}(\mathbf{d}), \\ \mathcal{B C}_{n} & \text { if } d_{0}=b_{0}(\mathbf{d}), \text { defined in Proposition } 1.18, \\ \mathcal{A C}_{n}-\left(\mathcal{C}_{n} \cup \mathcal{B C}_{n}\right) & \text { if } b_{0}(\mathbf{d})<d_{0}<h_{0}(\mathbf{d}), \\ \mathcal{H C}_{n} & \text { if } d_{0}=h_{0}(\mathbf{d}), \text { defined in Corollary } 3.5, \\ \mathcal{E}_{n} & \text { if } h_{0}(\mathbf{d})<d_{0} \leq \sum_{i=1}^{n-1} d_{i} .\end{cases}
$$

Moreover, as a function of $d_{0}, D_{0}\left(d_{0}, \mathbf{d}\right)$ is continuous, strictly increasing on $\left[D, b_{0}(\mathbf{d})\right]$ and strictly decreasing on

$$
\left[b_{0}(\mathbf{d}), \sum_{i=1}^{n-1} d_{i}\right] .
$$

Proof. The breakdown of where points on the ray $\left[D, \sum d_{i}\right]$ lie follows from Proposition 1.17 (for $\mathcal{C}_{n}$ and $\mathcal{B C}_{n}$ ), Corollaries 1.16 (for $\mathcal{A C}_{n}$ ), 3.5 (for $\mathcal{H C}_{n}$ ), and 4.6 (for $\mathcal{E}_{n}$ ). Continuity follows from Propositions 3.7 and 4.9, monotonicity from Propositions 2.3 and 4.9 .

Corollary 4.11. For fixed positive real numbers $d_{1}, \ldots, d_{n-1}$, among all hyperbolic $n$-gons with $n-1$ sides of these lengths, the area is maximized by the semicyclic ones with final side length greater than $\max \left\{d_{i}\right\}$.

Proof. The minimal possible final side length of a hyperbolic $n$-gon is

$$
D_{0}=\max \left\{0, d_{i_{0}}-\sum_{i \neq i_{0}} d_{i}\right\}
$$

where $d_{i_{0}}$ is maximal among the $d_{i}$. Let us take $\mathbf{d}=\left(d_{1}, \ldots, d_{n-1}\right) \in$ $\left.(0, \infty)^{n-1}\right)$, and consider the values of the area function $D_{0}$ at points $\left(d_{0}, \mathbf{d}\right)$ for $d_{0} \in\left[D_{0}, \sum_{i=1}^{n-1} d_{i}\right]$. These are maximized at $d_{0}=b_{0}(\mathbf{d})$ since, by Corollary 4.10, this point maximizes the value of $D_{0}\left(d_{0}, \mathbf{d}\right)$ for 
$d_{0} \geq D=\max \left\{d_{i}\right\}$, and Propositions 2.3 and 4.9 imply that $D_{0}\left(d_{0}, \mathbf{d}\right)$ increases for $D_{0} \leq d_{0} \leq D$.

The point $\left(b_{0}(\mathbf{d}), \mathbf{d}\right) \in \mathcal{B C}_{n}$ represents a semicyclic $n$-gon with $n-1$ sides of length $d_{1}, \ldots, d_{n-1}$ (see Corollary 4.10 above). Its area maximizes areas of all cyclic, horocyclic or equidistant $n$-gons with $n-1$ sides of length $d_{1}, \ldots, d_{n-1}$, since $D_{0}$ is symmetric. But, by Schlenker's Theorem C [12, pp. 2159-2160], the area of all hyperbolic $n$-gons with a fixed side length collection is maximized by the cyclic, horocyclic, or equidistant $n$-gon with that side length collection. Our result follows.

5. Degenerations. This section describes the closure $\overline{\mathcal{A C}}_{n}$ of $\mathcal{A C}$. in $[0, \infty)^{n}$. By Corollary $3.5, \mathcal{A C}_{n} \cup \mathcal{H C}_{n}$ is the closure of $\mathcal{A C}_{n}$ in $(0, \infty)^{n}$. It thus remains to describe limits of Cauchy sequences in $\mathcal{A C}_{n}$ that have some entries approaching 0 . The limit of most such sequences lies in copies of $\mathcal{A C}_{m}$, for $m<n$.

Lemma 5.1. Fix $n \geq 3$. For each $m$ with $0<m<n$, let $\mathcal{I}_{m, n}$ be the collection of $I=\left(i_{0}, \ldots, i_{m-1}\right) \in \mathbb{N}^{m}$ such that $0 \leq i_{1}<i_{2}<$ $\cdots<i_{m} \leq n-1$. For such an $m$ and $I$, define $\phi_{I}: \mathbb{R}^{m} \rightarrow \mathbb{R}^{n}$ by $\phi_{I}\left(x_{0}, \ldots, x_{m-1}\right)=\left(y_{0}, \ldots, y_{n-1}\right)$ where, for $0 \leq i \leq n-1$,

$$
y_{i}= \begin{cases}x_{i_{j}} & \text { if } i=i_{j}, \text { for some } 0 \leq j \leq m-1, \\ 0 & \text { otherwise. }\end{cases}
$$

Taking $\Delta=\{(r, r) \mid r \geq 0\}$, the closure of $\mathcal{A C}_{n}$ in $[0, \infty)^{n}$ is:

$$
\mathcal{A C}_{n} \sqcup \mathcal{H C}_{n} \sqcup\left(\bigsqcup_{\substack{3 \leq m<n \\ I \in \mathcal{I}_{m, n}}} \phi_{I}\left(\mathcal{A C}_{m} \cup \mathcal{H C} \mathcal{C}_{m}\right)\right) \sqcup\left(\bigcup_{I \in \mathcal{I}_{2, n}} \phi_{I}(\Delta)\right) .
$$

Call this union $\overline{\mathcal{A C}}_{n}$. Any two $\phi_{I}(\Delta)$ intersect only at $(0, \ldots, 0)$. The closure of $\mathcal{C}_{n}$ has an entirely analogous description, but with each instance of $\mathcal{A C}$ above replaced by $\mathcal{C}$ and each instance of $\mathcal{H C}$ replaced by $\mathcal{B C}$.

Proof. For a sequence in $\mathcal{A C}_{n}$ converging in $[0, \infty)^{n}$ to a point $\left(d_{0}, \ldots, d_{n-1}\right) \notin \mathcal{A C}_{n} \cup \mathcal{H C}_{n}$ at least one entry $d_{i}$ is equal to 0 . Note that, if all $d_{i}=0$, then trivially, $\left(d_{0}, \ldots, d_{n-1}\right) \in \phi_{I}(\Delta)$ for any $I \in \mathcal{I}_{2, n}$, so we may assume this does not hold. Let $d_{i_{0}}, d_{i_{1}}, \ldots, d_{i_{m}}$ 
be the non-zero entries, ordered so that $i_{0}<i_{1}<\cdots<i_{m}$, and let $I=\left(i_{0}, \ldots, i_{m}\right) \in \mathcal{I}_{m, n}$.

By construction, $\left(d_{0}, \ldots, d_{n-1}\right) \in \phi_{I}\left(\left(\mathbb{R}^{+}\right)^{m}\right)$, and it is not hard to see that $m$ and $I$ are unique with this property. The key observation is that, for each $j$ between 0 and $m-1$, by definition of $\mathcal{A C}_{n}$ (recall Corollary 1.16) and continuity of the hyperbolic sine:

$$
\sinh \left(d_{i_{j}} / 2\right) \leq \sum_{k \neq j} \sinh \left(d_{i_{k}} / 2\right) .
$$

It follows in short order that $m>1$. If $m=2$, then $d_{i_{0}}=d_{i_{1}}$, i.e., $\left(d_{0}, \ldots, d_{n-1}\right) \in \phi_{I}(\Delta)$, and if $m \geq 3$, then $\left(d_{0}, \ldots, d_{n-1}\right) \in$ $\phi_{I}\left(\mathcal{A C}_{m} \cup \mathcal{H C}_{m}\right)$. Moreover, $\left(d_{0}, \ldots, d_{n-1}\right)$ lies in $\phi_{I}\left(\mathcal{H C}_{m}\right)$ if and only if equality holds for some $j$ above (recall Corollary 3.5).

A similar argument using the definition of $\mathcal{C}_{n}$ (see Proposition 1.17) shows that a limit point of $\mathcal{C}_{n}$ outside $\mathcal{C}_{n}$ is in $\phi_{I}(\Delta)$ or $\phi_{I}\left(\mathcal{C}_{m} \cup \mathcal{B C} \mathcal{C}_{m}\right)$ for some $I$ in $\mathcal{I}_{2, n}$ or $\mathcal{I}_{m, n}$ for $m \geq 3$, respectively.

Finally, it is not difficult to show, for each $m \geq 3$ and $I \in \mathcal{I}_{m, n}$, that each point of $\phi_{I}\left(\mathcal{A C}_{m}\right)$ (or $\left.\phi_{I}\left(\mathcal{C}_{m}\right)\right)$ is approached by a sequence in $\mathcal{A C}_{n}$ (respectively, $\mathcal{C}_{n}$ ); and, moreover, that each point of $\phi_{I}(\Delta)$ is approached by a sequence in $\mathcal{C}_{n}$ for any $I \in \mathcal{I}_{2, n}$.

The parametrizations of $\mathcal{B C}_{n}$ and $\mathcal{H C}_{n}$ extend similarly to the closure of $\left(\mathbb{R}^{+}\right)^{n-1}$ in $\mathbb{R}^{n-1}$.

Lemma 5.2. For $n \geq 3$, the functions $b_{0}$ and $h_{0}$ on $\left(\mathbb{R}^{+}\right)^{n-1}$ from Propositions 1.18 and 3.5, respectively, extend continuously to $[0, \infty)^{n-1}$. For $3 \leq m<n$ and $I \in \mathcal{I}_{m-1, n-1}$ (as in Lemma 5.1), $b_{0}=b_{0} \circ \phi_{I}$ and $h_{0}=h_{0} \circ \phi_{I}$ on $\left(\mathbb{R}^{+}\right)^{m-1}$.

Proof. For $3 \leq m<n$ and $\mathbf{d}=\left(d_{1}, \ldots, d_{m-1}\right) \in\left(\mathbb{R}^{+}\right)^{m-1}$, the equation $\sum_{i=1}^{m-1} \theta\left(d_{i}, J\right)=\pi$, is uniquely solved by $J=b_{0}(\mathbf{d}) / 2>$ $\max \left\{d_{i}\right\} / 2$. Since $\theta(d, J)$ decreases in $J$ for any $d>0$, it follows that

$$
\sum_{i=1}^{n-1} \theta\left(d_{i}, J_{0}\right)>\pi>\sum_{i=1}^{m-1} \theta\left(d_{i}, J_{1}\right)
$$

for any $J_{0}$ and $J_{1}$ with $\max \left\{d_{i}\right\} / 2<J_{0}<b_{0}(\mathbf{d}) / 2<J_{1}$. Fixing $I \in \mathcal{I}_{m-1, n-1}$, it follows that values of the corresponding sum on a 
fixed sequence in $\left(\mathbb{R}^{+}\right)^{n-1}$ approach $\phi_{I}(\mathbf{d})$ greater than $\pi$ at all $J \leq J_{0}$ and less than $\pi$ for $J \geq J_{1}$. Therefore, values of $b_{0}$ on this sequence are eventually contained in $\left(2 J_{0}, 2 J_{1}\right)$.

The above implies that $b_{0}$ on $\left(\mathbb{R}^{+}\right)^{n-1}$ extends continuously by $b_{0} \circ \phi_{I}$ at $\phi_{I}(\mathbf{d})$. The proof for $h_{0}$ is similar. Let us also note that $b_{0}(\mathbf{d}) \rightarrow 0$ and $h_{0}(\mathbf{d}) \rightarrow 0$ if $\mathbf{d} \rightarrow 0$. This is because the value of either function is bounded above by $(n-1) \max \left\{d_{i}\right\}$ at $\left(d_{1}, \ldots, d_{n-1}\right)$, a side length of an $n$-gon whose other side lengths are the $d_{i}$.

It remains to consider limits with exactly one nonzero entry. Directly applying the definitions in this case implies that the extensions must satisfy:

$$
b_{0}(0, \ldots, 0, d, 0, \ldots, 0)=d=h_{0}(0, \ldots, 0, d, 0, \ldots, 0) .
$$

Arguing as above establishes that the so-defined extensions are continuous.

The radius function $J$ does not extend continuously to $\overline{\mathcal{A C}}_{n}$ since it blows up near $\mathcal{H C}_{n}$. Moreover, it is not hard to show that $\lim _{x \rightarrow 0} h_{0}(d, x)=d$, and, by consequence, that $(d, d, 0)$ (or indeed any point of any $\left.\phi_{I}(\Delta)\right)$ is a limit of horocyclic polygons. Because $\phi_{I}(\Delta)$ is also in $\overline{\mathcal{C}}_{n}$, it follows that $J$ does not have a well-defined limit on this set. It does on the remainder of $\overline{\mathcal{A C}}_{n}$, although .

Lemma 5.3. For any $n \geq 3, J: \mathcal{A C}_{n} \rightarrow \mathbb{R}^{+}$extends continuously to $\bigsqcup \phi_{I}\left(\mathcal{A C}_{m}\right)$, where $I$ runs over index sets in $\mathcal{I}_{m, n}$ with $2<m<n$, and for such $I$ that $J \circ \phi_{I}=J$. Here the left-hand $J$ acts on $\mathcal{A C}_{n}$ but the right-hand $J$ acts on $\mathcal{A C}_{m}$.

Proof. Since $J: \mathcal{A C}_{n} \rightarrow \mathbb{R}$ is symmetric, it suffices to consider sequences in $\mathcal{A C}_{n}$ converging to some $(\mathbf{d}, 0, \ldots, 0)$ for $\mathbf{d} \in \mathcal{A C}_{m}, 2<m<$ $n$, i.e., to restrict attention to $\phi_{I}\left(\mathcal{A C}_{m}\right)$, where $I=(0,1,2, \ldots, m-1)$. Moreover, we will assume that $\mathbf{d}$ has maximal first entry. Lemma 1.15 then implies that the equation $d_{0}=\ell^{n}\left(J, d_{1}, \ldots, d_{n-1}\right)$ holds on a neighborhood of $\mathbf{d}$ in $\mathcal{A C}_{m}$. We claim that, in fact, it holds on a neighborhood of $(\mathbf{d}, 0, \ldots, 0) \in \mathcal{A C}_{n}$.

The key fact is that one of $d_{0}=\ell^{n}\left(J, d_{1}, \ldots, d_{n-1}\right)$ or $d_{0}=$ $\ell^{s}\left(J, d_{1}, \ldots, d_{n-1}\right)$ holds at each point of any such neighborhood, where $J=J\left(d_{0}, \ldots, d_{n-1}\right)$. But, $\ell^{s}$ decreases in $J$ on $[D / 2, \infty)$, and 
$\ell^{s}(D / 2)<D$ for $D=\max \left\{d_{i}\right\}_{i=1}^{n-1}$, as calculated in the proof of Lemma 1.15. The claim follows.

Lemma 1.15 asserts that $J(\mathbf{d})>D / 2$, where $D=\max \left\{d_{i}\right\}_{i=1}^{m-1}$. Since $\ell^{n}$ increases in $J$ we have $\ell^{n}\left(J_{0}\right)<d_{0}<\ell^{n}\left(J_{1}\right)$ for any $J_{0}, J_{1}$ with $D / 2<J_{0}<J(\mathbf{d})<J_{1}$, taking $d_{0}$ here to be the initial entry of d. But, on inspecting the definition of $\ell^{n}$, it is clearly a continuous function of $\left(d_{1}, \ldots, d_{n-1}, J\right)$, on a neighborhood of $(\mathbf{d}, 0, \ldots, 0, J(\mathbf{d}))$, so the inequality above holds for all $\left(d_{0}, \ldots, d_{n-1}\right) \in \mathcal{A C}_{n}$ sufficiently near $(\mathbf{d}, 0, \ldots, 0)$. It follows that $J_{0}<J\left(d_{0}, \ldots, d_{n-1}\right)<J_{1}$ at such points.

Below, we show that the area function $D_{0}$ extends continuously to all of $\overline{\mathcal{A C}}_{n}$. Since the radius function $J$ extends continuously to

$$
\mathcal{A C}_{n} \cup \bigcup \phi_{I}\left(\mathcal{A C}_{m}\right)
$$

the diagonal functions $\ell_{i, j}$ of Corollary 1.21 do too, so by its definition in Corollary 1.23, $D_{0}$ does as well. Proposition 3.7 further handles the extension to $\mathcal{H C}_{n}$, so what we address below is the $\phi_{I}(\Delta)$.

Lemma 5.4. For any $n \geq 4$ and $0 \leq i_{0}<i_{1} \leq n-1$, let $I=\left(i_{0}, i_{1}\right)$. For any $d \geq 0$,

$\lim _{\mathbf{d} \rightarrow \phi_{I}(d, d)} \ell_{i, j}(\mathbf{d})= \begin{cases}0 & \text { if } i \text { and } j \text { are cyclically between } i_{0} \text { and } i_{1} ; \text { or } \\ d & \text { otherwise. }\end{cases}$

Here we say $i$ and $j$ are cyclically between $i_{0}$ and $i_{1}$ if either $i_{0} \leq i, j<$ $i_{1}$, or if each of $i$ and $j$ is less than $i_{0}-1$ ( taken to be $n-1$ if $i_{0}=0$ ) or at least $i_{1}$.

Proof. One simply observes that, for any cyclic polygon $\left\{x_{0}, \ldots, x_{n-1}\right\}$ with side length collection $\left(d_{0}, \ldots, d_{n-1}\right)$ near $\phi_{I}(d, d)$, there is a sequence of edges joining $x_{i}$ to $x_{j}$, such that all but at most one has length near zero; and there is one with length near $d$ if and only if $i$ and $j$ are not cyclically between $i_{0}$ and $i_{1}$. The result thus follows from the triangle inequality.

Corollary 5.5. For any $n \geq 3, D_{0}: \mathcal{A C}_{n} \rightarrow \mathbb{R}^{+}$extends continuously to $\overline{\mathcal{A C}}_{n}$, satisfying $D_{0} \circ \phi_{I}=D_{0}$ for any $I \in \mathcal{I}_{m, n}$ with $2<m<n$, and $D_{0} \equiv 0$ on $\phi_{I}(\Delta)$ for any $I \in \mathcal{I}_{2 \cdot n}$. 
Proof. For $A$ as in Lemma 1.22 it is straightforward to compute:

$$
\lim _{(x, y, z) \rightarrow(0,0,0)} A(x, y, z)=0=\lim _{(x, y, z) \rightarrow(d, d, 0)} A(x, y, z) .
$$

Inspecting the definition of $D_{0}$ in Proposition 2.3, one sees that its values on a sequence approaching $\phi_{I}(d, d)$ are sums of limits of the forms above.

Acknowledgments. The author thanks the anonymous referee for his or her careful reading and suggestions that have improved the paper.

\section{REFERENCES}

1. Stanko Bilinski, Zur Begründung der elementaren Inhaltslehre in der hyperbolishchen, Ebene. Math. Ann. 180 (1969), 256-268.

2. Martin R. Bridson and André Haefliger, Metric spaces of non-positive curvature, Grund. Math. Wissen. 319, Springer-Verlag, Berlin, 1999.

3. Jason DeBlois, The Delaunay tessellation in hyperbolic space, preprint, arXiv: 1308.4899 .

4. , The centered dual and the maximal injectivity radius of hyperbolic surfaces, Geom. Topol. 19 (2015), 953-1014.

5. Svetlana Katok, Fuchsian groups, Chicago Lectures in Mathematics, University of Chicago Press, Chicago, IL, 1992.

6. F. Miller Maley, David P. Robbins and Julie Roskies, On the areas of cyclic and semicyclic polygons, Adv. Appl. Math. 34 (2005), 669-689.

7. A.D. Mednykh, Brahmagupta formula for cyclic quadrilaterials in the hyperbolic plane, Sib. Elektr. Mat. Izv. 9 (2012), 247-255.

8. A.F. Möbius, Ueber die Gleichungen, mittelst welcher aus den Seiten eines in einen Kreis zu beschreibenden Vielecks der Halbmesser des Kreises und die Flache des Vielecks gefunden werden, Crelle's J. 3 (1828), 5-34.

9. Igor Pak, The area of cyclic polygons: Recent progress on Robbins' conjectures, Adv. Appl. Math. 34 (2005), 690-696.

10. John G. Ratcliffe, Foundations of hyperbolic manifolds, Grad. Texts Math. 149, Springer-Verlag, New York, 1994.

11. David P. Robbins, Areas of polygons inscribed in a circle, Amer. Math. Month. 102 (1995), 523-530.

12. Jean-Marc Schlenker, Small deformations of polygons and polyhedra, Trans. Amer. Math. Soc. 359 (2007), 2155-2189.

13. Wilson Stothers' geometry pages, www.maths.gla.ac.uk/wws/cabripages/ cabrio.html, in particular, see http://www.maths.gla.ac.uk/wws/cabripages/ hyperbolic/overview.html.

14. William P. Thurston, The geometry and topology of 3-manifolds, mimeographed lecture notes, 1979. 
15. Rolf Walter, Polygons in hyperbolic geometry 1: Rigidity and inversion of the $n$-inequality, preprint, arXiv:1008.3404v1.

16. _ Polygons in hyperbolic geometry 2: Maximality of area, preprint, arXiv: $1008.3821 \mathrm{v} 1$.

Department of Mathematics, University of Pittsburgh, Pittsburgh, PA 15260

Email address: jdeblois@pitt.edu 